\title{
Parental Alienation (Syndrome) - Eine ernst zu nehmende Form von psychischer Kindesmisshandlung
}

\author{
Wilfrid von Boch-Galhau
}

Eingegangen: 27. November 2017 / Angenommen: 24. März 2018 / Online publiziert: 13. April 2018

(c) Der/die Autor(en) 2018

\begin{abstract}
Zusammenfassung Bei der induzierten Eltern-KindEntfremdung (Parental Alienation) handelt es sich um eine bestimmte Form von psychischer Kindesmisshandlung, die im DSM-5, dem aktuellen Diagnostic and Statistic Manual der American Psychiatric Association (APA) unter der Diagnoseziffer V 995.51 child psycholocical abuse verortet ist. Induzierte ElternKind-Entfremdung, die nicht behandelt wird, kann $\mathrm{zu}$ traumatischen psycho-physischen Langzeitfolgen bei betroffenen Kindern führen. Dieser Tatsache wird in familiengerichtlichen Verfahren noch unzureichend Beachtung geschenkt. Der Artikel befasst sich zusammenfassend mit der Definition, der Symptomatik und den verschiedenen Schweregraden von Parental Alienation und beschreibt einige wichtige Entfremdungstechniken und mögliche psychosomatische und psychiatrische Folgen der induzierten Eltern-Kind-Entfremdung. Schließlich wird auf Präventions- und Interventionsprogramme hingewiesen, die inzwischen in einigen Ländern angewandt und evaluiert werden. Zwei Fallbeispiele aus der Praxis und ein ausführliches, internationales Literaturverzeichnis schließen die Arbeit ab.
\end{abstract}

Schlüsselwörter Induzierte Eltern-Kind-Entfremdung - Parental Alienation - Hochkonflikthafte Tren-

\footnotetext{
Diese Arbeit erschien im September 2016 in Kurzform in „Praxisleitfaden Kinderschutz in Kita und

Grundschule“, S. 79-84, Deutsche Kinderhilfe (Hrsg.), Wolters-Kluwer-/Carl-Link-Verlag, Köln. Veröffentlichung in ausführlicher Fassung erfolgt mit freundlicher Genehmigung des Verlages vom 21.10.2016.

Das ausführliche internationale Literaturverzeichnis finden Sie auf www.drvboch.de und www.pas-konferenz.de.

Dr. med. W. von Boch-Galhau $(\bowtie)$

Oberer Dallenbergweg 15, 97082 Würzburg, Deutschland praxis@drvboch.de
}

nung/Scheidung • Psychische Kindesmisshandlung • Psychische Langzeitfolgen · Interventionsprogramme

\section{Parental Alienation (Syndrome) - A serious form of child psychological abuse}

Summary Induced parental alienation is a specific form of psychological child abuse, which is listed in DSM-5, the current Diagnostic and Statistical Manual of the American Psychiatric Association (APA), under diagnostic code V 995.51 "child psychological abuse". Untreated induced parental alienation can lead to long-term traumatic psychological and physical effects in the children concerned. This fact is still not given sufficient attention in family court cases. The article gives a condensed overview of parental alienation, summarising its definition, the symptoms and the various levels of severity. It also describes some major alienation techniques and possible psychosomatic and psychiatric effects of induced parental alienation. Finally, attention is drawn to programmes of prevention and intervention now used and evaluated in some countries. The article concludes with two real-life examples from psychiatric practice, and a comprehensive list of international references.

Keywords Induced parent-child-alienation - Parental Alienation · High conflict separation/divorce - Child psychological abuse - Psychological long term consequences · Intervention programs

\section{Vorbemerkungen}

Seit 20 Jahren befasse ich mich als ErwachsenenPsychiater und Psychotherapeut mit dem Komplex Parental Alienation und Parental Alienation Syndrome/ Disorder und ich erlebe ständig das Leid betroffener erwachsener Scheidungskinder und betroffener aus- 
gegrenzter Mütter, Väter und Großeltern, die nach Trennung oder Scheidung den Kontakt zu ihren Kindern/Enkeln über viele Jahre oder ganz verloren haben. Derweil liefern sich Befürworter und Gegner dieses Konzeptes in Wissenschaft und Praxis erhebliche - teils ideologisch geprägte - Debatten [123, 149], ob es sich bei dem unzweifelhaft bestehenden Phänomen der induzierten Eltern-Kind-Entfremdung um ein „Syndrom“ handelt oder nicht, welchen Namen man ihm geben soll und ob es überhaupt existiert [20, 75].

Kritische Diskussionen sind wesentlich für den wissenschaftlichen Fortschritt. Problematisch ist allerdings, dass Reaktionen gegen PA(S) oft nicht auf wissenschaftlichen Argumenten und empirischen Forschungsergebnissen, sondern auf subjektiven Meinungen oder gar Ideologien beruhen. Bei den Kontroversen zwischen Befürwortern und Gegnern beziehungsweise Kritikern des PA(S)-Konzeptes gibt es noch erhebliche Missverständnisse und Vorbehalte. Zum Beispiel: Es gebe noch $\mathrm{zu}$ wenig empirische Forschung; PA(S) sei eine simplistische, pseudowissenschaftliche Theorie; keine „wissenschaftlich begründete Entität und eine Ideologie“; PA(S) sei nicht durch berufliche Organisationen, besonders nicht durch DSM-5 anerkannt; Es sei eine Erfindung von Richard A. Gardner und seiner Kollegen/-innen, bis hin zu ad hominem Angriffen, sie propagierten Pädophilie; Die Praxis der Interventionen sei eine „ThreatTherapie“ und „traumatisch“ für betroffene Kinder; PA(S) sei ein Gender-Thema, bei dem missbrauchende oder gewalttätige Väter protektiven Müttern mit Hilfe von Gerichten das Sorgerecht absprechen wollten; Vorwürfe des sexuellen Missbrauchs seien immer zutreffend und Kinder in ihrem erklärten Willen nicht manipulierbar. Dies sind einige Missverständnisse und grobe Fehlinformationen. Diese werden vor allem in der „Domestic-Violence“-, der „Child-Abuse-“ und der feministischen Literatur vorgetragen. Kritiker im deutschen Sprachraum sind zum Beispiel Salgo [129]; Salzgeber [130, 131]; Fegert [56-58]; Heiliger und Hack [82]; et al.

In USA zum Beispiel Faller [55]; Bruch [37]; jüngst: Clemente et al. [44-46]; et al. Die amerikanischen Arbeiten von Lorandos [106]; Kopetski [102]; Warshak [147, 151]; Rand [123]; Bernet und Baker [21]; Bernet [20]; Baker et al. [12] setzen sich mit diesen einseitigen und falschen Behauptungen zur Theorie und Praxis von Parental Alienation intensiv auseinander und widersprechen ihnen sehr deutlich. Auseinandersetzungen finden noch vorwiegend über die Rolle des sogenannten entfremdenden Elternteils und welche Interventionsmodelle angemessen sind, statt.

Die seit Jahren kontrovers diskutierte Schlüsselfrage ist, ob es bei Trennung oder Scheidung Väter und Mütter gibt, die ihr Kind dahingehend manipulieren, dass es den Kontakt zu dem anderen Elternteil endgültig ablehnt, ob dieses eine negative Wirkung auf die psychische Gesundheit und Entwicklung des
Kindes hat und welche Interventionen erfolgversprechend sind. Die Antworten auf diese Fragen haben praktische Bedeutung sowohl für das Familienrechtssystem als auch für „Mental Health Practitioners“, die mit betroffenen Scheidungsfamilien arbeiten.

Vor diesem Hintergrund versuche ich hier darzustellen, dass es sich bei der induzierten Eltern-KindEntfremdung (in der internationalen Fachwelt hat sich der englische Ausdruck „Parental Alienation“, also ohne „Syndrome“, mehr oder weniger durchgesetzt) um eine gravierende Form von psychischer Kindesmisshandlung handelt, die mit traumatischen psychophysischen Langzeitfolgen in der Persönlichkeit des Kindes und des späteren Erwachsenen verbunden sein kann [27, 96].

Parental Alienation: Eltern-Kind-Entfremdung - ist nach dem „Diagnostic and Statistical Manual“ DSM5 (der für USA maßgebliche Diagnose-Katalog) eine klinisch relevante Beziehungsstörung (parent-child relational problem) mit erheblichen Folgen für die betroffenen Kinder. Diese ist noch nicht überall offiziell unter dem Begriff Parental Alienation anerkannt. Daher hat die American Psychiatric Association (APA) den Begriff Parental Alienation noch nicht explizit in das DSM-5 aufgenommen. PA(S) sei außerdem nicht „im Kind“ angelegt und daher keine individuelle psychiatrische „Mental Condition“. Zwei neue und zwei alte Diagnosen („Child psychological abuse“, „Child affected by parental relationship distress“ (CAPRD), „Parent-child-relational-problem“ und „Induced delusional disorder") sind jedoch jetzt schon darin enthalten, die es Klinikern und psychologischen/ psychiatrischen Gerichtssachverständigen erlauben, Kinder und Jugendliche $\mathrm{zu}$ identifizieren und differentialdiagnostisch einzuordnen, die von Parental Alienation betroffen sind [24, 28]. Die weltweit gültige „International Classification of Diseases“ (ICD11) der Weltgesundheitsorganisation (WHO) wird erst in 2018 fertig gestellt sein. Da im Allgemeinen das Bestreben besteht, die Inhalte von DSM-5 und ICD11 aufeinander abzustimmen, ist anzunehmen, dass ähnliche Diagnosen für Parental Alienation dort integriert werden. Es wird zurzeit in einer Beta-Version von ICD-11 unter dem Begriff „Care-giver-child-relation-problem“ „Parental Alienation“ als Diagnose diskutiert.

Das Phänomen der induzierten Eltern-Kind-Entfremdung (Parental Alienation) wird seit mindestens 60 Jahren in der psychiatrischen Fachliteratur beschrieben [138], aber erst in den 1980- und 1990-er Jahren als solches benannt. Mindestens sechs Forscher oder Forscherteams identifizierten unabhängig voneinander Kinder aus Trennungs-/ Scheidungsfamilien, die von einem Elternteil ohne rationalen Grund vom anderen entfremdet waren: Wallerstein und Kelly [144, 145], Johnston und Roseby [90] und Johnston [89] sprechen von „Pathologischer Ausrichtung“ („pathological alignment“) und von „Umgangsverweigerung“ („visitation refusal“). Gardner 
[68] führte den Begriff „Parental Alienation Syndrome“ ein, den auch Rand [121, 122], Kopetski [100-102] und Kopetski et al. [103, 104] benutzten. Clawar und Rivlin $[42,43]$ sprechen von ,programmed and brainwashed children“. Kelly und Johnston [92] führten den Begriff das „Entfremdete Kind“ („The alienated child“) ein und Warshak [147] spricht von „Pathologischer Entfremdung“ (,pathological alienation“). Bernet [18] und Bernet et al. [22] verwenden die Begriffe „Parental Alienation Disorder“ und „Parental Alienation“. In der Folge wurde das Phänomen „Eltern-Kind-Entfremdung“ (Parental Alienation) bis heute von vielen internationalen Fachleuten aus Wissenschaft und Praxis weltweit beobachtet und beschrieben (zum Beispiel Hellblom-Sjögren [83]; Gardner et al. [72]; Bernet [19]; Lorandos et al. [108]; Foran et al. [65]; Whitcombe [155]; Paricard [117]; Hinterhofer et al. [86]; Broca und Odinetz, [34]; Hirigoyen [87]; Fernandez-Cabanillas [60]; Bensussan [15]).

In der aktuellen klinischen Literatur wird unterschieden zwischen Parental Alienation (ungerechtfertigte Ablehnung eines Elternteils aufgrund von Manipulation und Indoktrination des Kindes) und Estrangement (Gerechtfertigte Ablehnung eines Elternteils aufgrund einer tatsächlich stattgehabten Geschichte von Vernachlässigung, Misshandlung, sexuellem Missbrauch oder häuslicher Gewalt) [1, 10, 24, 25, 62, 63, 108, 132]. Die internationale Fachliteratur weist bis heute mehr als dreizehnhundert wissenschaftlich relevante Publikationen aus mehr als 45 Ländern zum Thema Parental Alienation, Parental Alienation Syndrome und angrenzenden Themen auf. Sie sind speziell auf der „Parental-Alienation-Datenbank“ [116] der medizinischen Fakultät der Vanderbilt-Universität in Nashville, TN, USA zu finden.

Die internationale Fachliteratur zeigt, dass die Prävalenzrate von elterlicher Entfremdung von Kindern und Jugendlichen in den USA etwa $1 \%$ beträgt [19, 81]. In Europa gibt es keine genauen Zahlen.

\section{Definition von PA(S)}

Das Konzept „Parental Alienation“ wird durch drei Elemente definiert [147]:

- Ablehnung oder Verunglimpfung eines Elternteils, die das Ausmaß einer Kampagne erreichen, das heißt andauernd und nicht nur als gelegentliche Episode.

- Die feindselige Ablehnungshaltung ist irrational und die Entfremdung ist nicht eine angemessene Reaktion auf das Verhalten des abgelehnten Elternteils. Sie beruht nicht auf tatsächlich gemachten negativen Erfahrungen mit dem zurückgewiesenen Elternteil.

- Sie ist Teilresultat des Einflusses des entfremdenden Elternteils [und/oder anderer wichtiger Bezugspersonen].

- Wenn eines dieser drei Elemente fehlt, kann nicht von PA oder PAS gesprochen werden.

\section{Symptomatik bei PA(S)}

- Unbegründete Zurückweisungs- und Verunglimpfungskampagne

- Absurde Rationalisierungen (ungerechtfertigte, absurde Begründungen für Ablehnungshaltung)

- Fehlen von normaler Ambivalenz (Idealisierung des einen Elternteils und Verteufelung des anderen, Schwarz-Weiß-Denken)

- Reflexartige Parteinahme für den programmierenden Elternteil

- Ausweitung der Ablehnung auf die gesamte Familie und das Umfeld des zurückgewiesenen Elternteils

- Das Phänomen der „eigenen Meinung“ (Betonung der „eigenen Meinung“ und des „eigenen Willens“)

- Verleugnung von Schuldgefühlen über die Grausamkeit gegenüber dem entfremdeten Elternteil (Entfremdeter Elternteil wird nach außen „eiskalt“ und ohne Gefühlsregung zurückgewiesen)

- Übernahme „geborgter Szenarien“ (gleiche Vorwürfe wie diese der entfremdende Elternteil vorbringt)

- Zur Validierung: Rueda [127]; Burrill [38]; Baker und Darnall [8, 9]; Hachenberg [80]; Saini et al. [128].

Differenzierung von PA(S) auf einem Kontinuum von drei Schweregraden, die jeweils spezielle Behandlungstechniken erfordern

In leichten Fällen [49] widersetzt sich das Kind, Kontakt mit dem außerhalb lebenden Elternteil (ET) aufzunehmen, jedoch genießt es diesen, sobald die Verbindung hergestellt ist. Es kann sich noch von den Abwertungen des entfremdenden ET gegenüber dem anderen distanzieren.

In mittelschweren Fällen [157] mit starker Ausprägung der Symptome und bereits erheblichen Umgangs- und Übergabeproblemen: Das Kind wehrt sich hartnäckig gegen Kontakt, es geht aber darauf ein, wenn dieser erst einmal zustande kommt und der entfremdende ET abwesend ist.

Bei PA(S) in der schweren Form [150] lehnt ein Kind den Kontakt zu einem ET (Vater oder Mutter), mit dem es zuvor eine liebevolle Beziehung und Bindung hatte, irrational und ohne objektiven Grund radikal $\mathrm{ab}$, weil es ein falsches, negatives Elternbild verinnerlicht hat. Die Ablehnungshaltung und der Grad der Negativität unterscheiden sich von der leichten und mittelschweren Form erheblich. Das Kind äußert eine extrem polarisierte Sichtweise seiner Eltern (Schwarz/Weiß). In diesem Fall ist das Familiengericht in Zusammenarbeit mit einem speziell ausgebildeten psychologischen/psychiatrischen Sachverständigen/ Therapeuten die letzte Autorität, die den Entfremdungsprozess (in der Regel durch Sanktionen oder durch glaubhafte Ankündigung, gegebenenfalls Realisierung von Sorgerechtstransfer) unterbrechen oder endgültig besiegeln kann (durch passives Zuwarten: 
„Wenn das Kind nicht will, kann man nichts machen.") [103, 151, 152].

Die Diagnose und der Schweregrad von PA(S) werden anhand des kindlichen Verhaltens festgestellt und nicht aufgrund des Ausmaßes der Manipulation, der das Kind ausgesetzt ist. Eine sorgfältige Diagnostik [32, 134] des gesamten Familiensystems und die Identifizierung der manipulierenden Person(en) sind unabdingbar. Auch die Rolle des sogenannten entfremdeten Elternteils und dessen Anteile am Entfremdungsprozess müssen genau abgeklärt werden, um Fehldiagnosen zu vermeiden.

PA(S) ist nicht „Umgangsvereitelung“ oder ,jedwede Art von Kontaktverweigerung“ eines Kindes gegenüber dem außerhalb lebenden Elternteil bei Trennung/Scheidung - wie manche Kritiker meinen (zusammenfassend Gödde [76]) -, sondern eine psychiatrisch relevante Beziehungsstörung zwischen Kind und Eltern aufgrund einer psychischen Traumatisierung [69-71, 147, 151, 152]. Die kognitive und emotionale Ebene und das Verhalten des Kindes werden durch die Misshandlungssituation beeinträchtigt. Im Unterschied zu anderen, zum Beispiel - psychodynamischen - Erklärungsversuchen von kindlicher Kontaktverweigerung [64] liegt bei PA(S) regelmäßig eine massive Umgangsbehinderung/-vereitelung und/oder Manipulation/Indoktrination des Kindes vor.

Die aktive Manipulation erfolgt - bewusst oder unbewusst - durch den betreuenden Elternteil und/ oder andere Bezugspersonen (nicht geschlechtsspezifisch!), von denen das Kind abhängig ist. Bei den manipulierenden Bezugspersonen lassen sich psychische Auffälligkeiten identifizieren, wie schwere narzisstische und/oder Borderline-Persönlichkeitsstörungen [87, 100, 101, 135], traumatische Kindheitserfahrungen [23, 26], paranoide Verarbeitung der Scheidungskrise und/ oder Psychosen [69, 70, 91].

Einstellung und Verhalten von Scheidungsbegleitern spielen im weiteren Verlauf von induzierten Entfremdungsprozessen eine große Rolle [2, 3, 81].

\section{Wichtige Entfremdungstechniken bei PA(S)}

Baker und Darnall [8] identifizierten in einer Studie mit 97 Erwachsenen, die sich als Opfer von ElternKind-Entfremdung beschrieben, 66 verschiedene Entfremdungsstrategien; 11 davon wurden von mindestens $20 \%$ der Probanden ihrer Stichprobe erwähnt. Bei der Induktion von PA(S) sind unter anderem irrationale Abwertung, realitätsverzerrende Negativdarstellung des anderen Elternteils, Umgangsboykott, Kontaktunterbrechung, gezielte Fehlinformationen, suggestive Beeinflussung und/oder Vermittlung von verwirrenden Doppelbotschaften von Bedeutung. Bisweilen wird direkte psychische (wie Androhung von Liebesentzug und/oder Suizid) und körperliche Gewalt (wie Schläge, Einsperren) gegen die Kinder eingesetzt [8, 9]. Zwei Dokumentarfilme von G. Gebhard
„Victims of Another War - The Aftermath of Parental Alienation“ [73] und „Sarah Cecilie“ [74] ${ }^{1}$ zeigen das Problem aus der Sicht der ehemals entfremdeten Kinder. Ich empfehle, sich diese beiden Filme anzusehen (siehe Links in den Literaturangaben $\mathrm{zu}$ Gebhard). Der ohnehin bestehende Loyalitätskonflikt des Kindes wird verschärft. Angst, emotionale Abhängigkeit, Unterwerfung, „Gefügigmachen“ des Kindes und dadurch angstbedingte Identifikation mit dem Entfremder spielen bei der Entstehung der Symptomatik eine wichtige Rolle [4-6, 41]. Eine ähnliche Psychodynamik findet sich beim Stockholm-Syndrom bei Geiselnahmen.

Lowenstein [110] erläutert in einem eigenen Kapitel seines Buches das Stockholm-Syndrom im $\mathrm{Zu}$ sammenhang mit dem bekannten österreichischen „Entführungsfall Natascha Kampusch“ und zeigt die Verwandtschaft zum Parental Alienation (Syndrome) auf. Auch bei Sektensystemen [5, 141] greifen ähnliche Mechanismen. Manche Fälle von PA(S) der schweren Form zeigen in ihrer Dynamik Gemeinsamkeiten mit dem Münchhausen-by-Proxy-Syndrom, einer Störung, bei der Eltern an ihren Kindern Krankheitssymptome künstlich erzeugen oder verstärken [54, 120]. Die Kinder sind auf Hilfe von außen angewiesen, um dieser pathologischen Situation zu entkommen.

\section{Psychiatrische und psychosomatische Folgen der PA(S)-Induktion für betroffene Scheidungs- kinder}

Die Erzeugung von PA(S) wird von verschiedenen internationalen Autoren wie etwa von Gardner, [69, 70], Kelly und Johnston [92], Deegener und Körner [50], Hirigoyen [87], et al. als psychische Kindesmisshandlung angesehen.

Rechtlich gesehen ist die Erzeugung von PA(S) als psychische Kindeswohlgefährdung durch missbräuchliche Ausübung der elterlichen Sorge unter Ausnutzung des Abhängigkeitsverhältnisses des Kindes einzuordnen [97, 114, 115, 136]. Das wird von einigen Kritikern des PA(S)-Konzeptes nicht ausreichend berücksichtigt und das Problem auf den „Elternkonflikt“ und/oder auf den „Loyalitätskonflikt“ des Kindes bei Trennung und Scheidung reduziert.

Bei Kindern und Jugendlichen, die mit wiederkehrenden massiven Ehekrisen ihrer Eltern, aggressiven Auseinandersetzungen und traumatischen Trennungen und Scheidungen konfrontiert werden, können als Folge dieser chronisch diffusen Belastungen Persönlichkeitsentwicklungsstörungen auftreten ([59], Adverse Childhood Experiences). Bei 70-90\% der Borderline-Störungen im Erwachsenenalter konnten retrospektiv traumatische Belastungen in der Kindheit festgestellt werden [158].

1 Sie können alle Versionen des Films durch die Action Against Abduction (Webseite auf https://www.youtube.com/user/ PACTonline einsehen). 
Bei PA(S)-Fällen der schweren Form kommt es zum langfristigen, nicht selten auch zum endgültigen Beziehungs- und Kontaktabbruch zwischen Kind und Elternteil, manchmal auch zwischen Geschwistern mit den damit verbundenen pathologischen Folgen $[13,118]$. Die psychische Traumatisierung des PA(S)Kindes, des hinterbliebenen Elternteils und anderer naher Verwandter (z. B. Großeltern) wird selten angemessen berücksichtigt [98, 99]. Derart traumatisierte Menschen finden sich später häufig mit erheblichen psychischen, psychosomatischen und psychiatrischen Problemen in nervenärztlichen und/oder psychotherapeutischen Fachpraxen und Kliniken wieder [29, 36, 94].

Ein konsistentes Ergebnis der Scheidungsforschung besagt, dass der primäre negative Aspekt der elterlichen Scheidung ein mit ihr einhergehender Verlust eines Elternteils für das Kind ist. Die sich aus fehlender Verfügbarkeit der Mutter oder des Vaters für das Kind ergebenden Konsequenzen sind in der Literatur weitreichend beschrieben [84, 145].

Hummel [88] beschreibt in ihrem Buch, warum Väter besonders häufig Opfer von induzierter ElternKind-Entfremdung werden. Im Kapitel „Entfremdete Kinder" wird von der Autorin - gut recherchiert - der eindrucksvolle PA(S)-Fall „Timo“ ein Lehrstück für scheidungsbegleitende Professionen dargestellt.

Die Induktion von PA(S) führt zu einer Verwirrung des Kindes in der Selbst- und Fremdwahrnehmung und $\mathrm{zu}$ einer tiefen Selbstentfremdung. Das Kind verlernt, den eigenen Gefühlen und der eigenen Wahrnehmung zu trauen. In seiner Abhängigkeit ist es auf das Wohlwollen des programmierenden und fremdbestimmenden Elternteils angewiesen. Es verliert das Gefühl für die Realität und für seine eigene Kontur. Die eigene Identität wird verunsichert und brüchig. Negative Selbsteinschätzung oder grandiose Selbstüberschätzung, Selbstwertmangel und tiefe Unsicherheit sind die Folgen. Es können sich spezifische Persönlichkeitsstörungen im Sinne von F. 60 der ICD10, Essstörungen, Suchterkrankungen, Posttraumatische Belastungsstörungen und andere psychische und psychosomatische Krankheiten entwickeln [142]. Das Selbst und der Kern des betroffenen Kindes, insbesondere Teile des biografischen Selbst und der Herkunft, werden durch die fremdbestimmte, aktive Zurückweisung, Negierung und realitätsverzerrte Negativbesetzung eines ursprünglich geliebten Elternteils tiefer beschädigt, als durch den Verlust an sich (wie etwa beim Todesfall). Beides - massive Schuldgefühle und der Elternanteil an der eigenen Person - müssen verdrängt bzw. abgespalten werden. Es fehlt eine stabile Verwurzelung im familiären Herkunftssystem des abgespaltenen Elternteils. Daraus können sich weitere langfristige Entwicklungs- und Beziehungsprobleme ergeben, die zum Teil transgenerational weitergegeben werden [77, 113, 146].

Die psychische Misshandlung ist schwer $\mathrm{zu}$ identifizieren, weil sie nicht mit einer Schädigungsabsicht in Erscheinung tritt. Mit ihren langfristigen psychopathologischen Auswirkungen ist sie aber - wie andere Formen der Misshandlung auch - keinesfalls zu tolerieren. Die Kinder müssen davor geschützt werden.

Analog zur Frage der Strafmündigkeit ist bei der Einschätzung des angeblichen Kindeswillens $\mathrm{zu}$ berücksichtigen, ob vom Entwicklungsstand her überhaupt von einer freien Willensentscheidung auszugehen ist und/oder ob der scheinbar „autonome Kindeswille“ nicht auf Manipulationen zurückgeht (Phänomen der „eigenen Meinung“ als Symptom bei PA(S)). Vor dem Hintergrund der Forschung über entwicklungspsychologische Zusammenhänge und systemische Komponenten, wie Loyalitätskonflikt und die maligne Nachscheidungsdynamik [112] sowie über Gedächtnis und Suggestibilität von Kindern durch Einflüsse Erwachsener, durch soziale Einflüsse und durch Zwangseinflüsse [39, 105, 119] und vor dem Hintergrund der Erfahrungen mit Beeinflussung von Kindern und Jugendlichen in Sektenund ideologischen Systemen [5, 141] erhält die Frage von geäußertem Kindeswillen und von kindlicher Erinnerung im Zusammenhang mit der Abklärung von $\mathrm{PA}(\mathrm{S})$, insbesondere von $\mathrm{PA}(\mathrm{S})$ und Vorwürfen des sexuellen Kindesmissbrauchs bei Trennungs- und Scheidungskonflikten eine besondere Bedeutung.

Um schwerwiegende Fehlentscheidungen (besonderes Aufsehen erregten in Europa, zum Beispiel in Deutschland die "Wormser Missbrauchsprozesse“ [137] und in Frankreich: „l'Affaire d'Outreau“ [14, 51]) für Kinder und Eltern im Zusammenhang mit strafrechtlichen Konsequenzen und mit Umgangs- und Sorgerechtsbeschlüssen zu vermeiden ist differentialdiagnostisch in letzterem Fall sehr sorgfältig zu unterscheiden zwischen a) realem sexuellem Missbrauch b) „Missbrauch des Missbrauchs“ als Strategie oder Pathologie (zum Beispiel Projektion von sexuellen Phantasien nach traumatischen Missbrauchserlebnissen als Kind auf den späteren Partner; paranoide Verarbeitung von Trennung und Scheidung; Psychosen) und c) Unzutreffenden sexuellen Missbrauchsvorwürfen bei PA(S) [16, 17, 137, 143].

Trotz der Bedeutung von Parental Alienation sowohl für „Mental Health“ als auch „Legal Professionals" und trotz seiner Akzeptanz durch Hunderte von Gerichtsurteilen weltweit, wird dieses Phänomen nach wie vor von einigen Kollegen abgelehnt. Dies führt immer noch zu problematischen Empfehlungen in familiengerichtlichen Gutachten und Gerichtsentscheidungen, das heißt: Entfremdende Eltern erhalten - ohne Auflagen - das alleinige Sorgerecht, entfremdete werden vom Umgang ausgeschlossen, damit das Kind zur angeblichen Ruhe komme. Dies ist jedoch eine „trügerische Ruhe, die die kindliche Entwicklung sogar gefährdet“ (Klenner [95], unter Berufung auf John Bowlby, [30, 31]: „Phasen der Auflehnung Verzweiflung - Resignation - Loslösung/Ablehnung“, 
im Sinne einer reaktiven Depression und psychischen Deprivation im Kindesalter).

\section{Exkurs: Ein Beispiel aus juristischer Sicht anhand eines Urteils zum Umgangsrecht in Deutschland (siehe Heuchemer, M. O. [85], in: Familie und Recht 7: 368-372)}

In seinem Artikel „Kardinalfehler der Rechtsprechung im Umgangsrecht - Rechtsfehler, Rechtsverletzungen und die (In-)Effizienz prozess- und materialrechtlicher Korrekturmechanismen im Instanzenzug am Beispiel der Eltern-Kind-Entfremdung (Parental Alienation [Syndrom]) “ beschäftigt sich Heuchemer äußerst kritisch mit dem jahrelangen gerichtlichen Umgangsausschluss eines Vaters mit seinem Sohn zwischen dem 11. und 17. Lebensjahr, ohne rechtlich nachvollziehbaren Grund. Dies geschah aufgrund einer fragwürdigen Gutachterbewertung der Situation eines entfremdeten Jungen, die sich die Gerichte durch sämtliche Instanzen bis zum Europäischen Gerichtshof (EGMR) in Straßburg zu Eigen machten. Die Schlussfolgerungen, die die Gutachter zogen: Ein Umgang stehe dem erklärten Willen des Kindes entgegen, es werde „traumatisiert“, wenn gegen seinen Willen ein Umgang durchgesetzt werde und es müsse „zur Ruhe kommen".

Nach Einschätzung von Heuchemer, der sich auf eine Fülle von Forschungsergebnissen aus den Bereichen Psychiatrie, Kinderpsychiatrie und Psychosomatik der letzten Jahre stützt, wird das Kind jedoch gerade durch diese Gerichtsentscheidungen einem „fortgesetzten psychischen Kindesmissbrauch, verbunden mit einer erheblichen Traumatisierung" ausgesetzt (S. 370). Es handle sich um eine gutachterliche Falschdeutung mit der Folge gravierender gerichtlicher Fehlentscheidungen hinsichtlich der Kurz- und Langzeitfolgen von Eltern-Kind-Entfremdung.

Ich empfehle dem Leser, diesen Artikel zu lesen, um sich ein eigenes Bild von der unbefriedigenden aktuellen Situation - vor allem in Deutschland - zum Thema Eltern-Kind-Entfremdung (Parental Alienation) zu machen.

\section{Internationale Entwicklungen}

Parental Alienation ist ein weltweites Phänomen, das sich in empirischen Studien in verschiedenen Ländern nachweisen ließ [52] und das sich in mehr als 600 Gerichtsentscheidungen widerspiegelt, zum Beispiel in USA und Kanada [19, 107]; im brasilianischen Gesetz über Parental Alienation/Law 12318 von 2010 [35]; in Gesetzen in einigen weiteren südamerikanischen Staaten [52] sowie in Urteilen des Europäischen Gerichtshofes für Menschenrechte (EGMR) [European Court of Human Rights (ECHR)] in Straßburg, zum Beispiel Sommerfeld ./. Deutschland (2003); Koudelka und Zavrel ./. Tschechische Repulik (2006 und 2007); Plasse-Bauer ./. Frankreich (2006); Minecheva
./. Bulgarien (2010); Bordeiana ./. Moldavien (2011) und andere [53]; in Frankreich durch das nationale Berufungsgericht [47] und in Oberlandesgerichtsentscheidungen verschiedener europäischer Länder, wie Deutschland, Italien, Schweiz, Frankreich, Schweden, England, Spanien, Rumänien und andere [19].

In einer australischen Metaanalyse von Templer et al. (Sept. [140]): Recommendations for best practice in response to parental alienation: findings from a systematic review, Journal of Family Therapy 00: 1-19 wurden 10 peer reviewed Arbeiten aus dem englischen Sprachraum aus den Jahren 1990 bis 2015 überprüft. Dabei fanden die Autoren, dass eine Änderung des Sorgerechtes (custodial changes) oder des Wohnortes des Kindes (residential arrangements) $\mathrm{zu}$ Gunsten des entfremdeten Elternteils effektiv sind für eine Verbesserung von Parental-Alienation-Fällen. Psychoedukation und spezielle systemische Familientherapie für alle Familienmitglieder ist dabei wirksam, um die familiäre Verbindung und Funktionsfähigkeit wieder aufzubauen. Eine koordinierte Herangehensweise von therapeutischen Interventionen und gerichtlichen Maßnahmen (mit Sanktionen bei unkooperativen Eltern) ist wesentlich um Parental-Alienation-Fälle zu lösen.

Diese Maßnahmen eignen sich nicht für Fälle, bei denen „Estrangement“ vorliegt, also der so genannte entfremdete Elternteil als echter Kindesmisshandler identifiziert wurde.

Außerdem fanden die Autoren, dass es für Kinder weniger schädlich sei, von dem entfremdenden Elternteil getrennt zu werden, als den Entfremdungsprozess unbeeinflusst weiter laufen zu lassen. Psychologische Betreuung für alle Familienmitglieder während dieser Interventionsphase sei erforderlich.

Keine der überprüften Studien empfahlen darauf $\mathrm{zu}$ warten, dass die „Entfremdung“ sich von alleine auflöse oder dass man Kinder entscheiden lassen solle, welcher Elternteil das Sorgerecht haben oder wo sie wohnen sollten.

\section{Best Practise in Deutschland: Die Cochemer Praxis}

Eine, seit 1993 im Gerichtsbezirk Cochem, RheinlandPfalz, erfolgreich praktizierte Form interdisziplinärer Zusammenarbeit, die in Deutschland als „Cochemer Praxis“ auch von der Politik wahrgenommen wird, erscheint als eine effektive Möglichkeit, der Entwicklung von $\mathrm{PA}(\mathrm{S})$ und den damit verbundenen sozialen, medizinisch-psychologischen und ökonomischen Folgen vorzubeugen. Die Arbeitsweise in Cochem beruht im Wesentlichen auf einer schnellen Terminierung von Familiensachen und dem Prinzip der „Konfliktlösung durch multiprofessionelle Vernetzung“ und der „Verordneten Kooperation im Familienkonflikt als Prozess der Einstellungsänderung" aus Sicht einer psychologischen Sachverständigen [67] und aus Sicht eines 
ehemaligen Familienrichters [126]. Die Cochemer Arbeitsweise ist aus der Praxis entstanden und basiert theoretisch auf der klassischen sozialpsychologischen Einstellungsforschung und der bekannten DissonanzTheorie (Festinger) [61]. Die Methode hat die Deeskalation des Elternkonfliktes durch Veränderung von einer „Gewinner-Verlierer-Einstellung“ bei Eltern und Professionellen und die Wiederherstellung der Eltern-Autonomie und -verantwortung auf der Basis des Schutzes sowohl der Rechte der Kinder als auch der Rechte der Eltern zum Ziel. Sie setzt ein hohes Maß an Erfahrung und Qualifikation der beteiligten interdisziplinären Berufsgruppen in der Arbeit mit hochstrittigen Scheidungsfamilien voraus. Es gibt inzwischen verschiedene ähnlich ausgestaltete Interventionsprogramme in Europa [33, 111]. Im deutschsprachigen Raum (vor allem in Österreich und der Schweiz) wird seit Jahren ein Kooperationsverfahren von Psychologie und Recht (Collaborative Law) ähnlich einem Mediationsverfahren - angeboten.

\section{Präventions- und Interventionsprogramme}

Hat sich erst einmal eine hochgradige Ablehnungshaltung beim Kind fixiert, ist es schwierig, eine geeignete Hilfe und Intervention einzuleiten. Viele Eltern, aber auch Jugendamtsmitarbeiter, Richter, Therapeuten und Gerichtssachverständige resignieren bei solchen unlösbar erscheinenden Fällen und raten zum Abwarten, bis das Kind eines Tages von alleine den Kontakt zum abgelehnten Elternteil aufnimmt. Hier gehen die Meinungen von Fachleuten sehr auseinander. Das unten erwähnte Beispiel in Punkt A zeigt, wie solche Fälle häufig ausgehen.

In USA, Kanada, England, Australien, Südafrika, Mexiko und Spanien sind psychologische Interventionsprogramme in Anwendung, die hier nur exemplarisch erwähnt werden können. Siehe zum Beispiel „Therapeutic interventions for children with parental alienation syndrome“ [70]; „The Psychological Effects and Treatment of the Parental Alienation Syndrome“ und „Parental Alienation: How to understand and address parental alienation resulting from acrimonious divorce or separation“ [109, 110]; „Family bridges: Using insights from social science to reconnect parents and alienated children“ [148]; „Commentary on ,Family bridges ...“ “ [93]; „Helping alienated children with ,Family bridges ...“ [154]; „When a child rejects a parent: Tailoring the intervention to fit the problem“ [66]; „Toxic divorce: A workbook for alienated parents“ [124]; „The psychosocial treatment of parental alienation“ [48]; „A Family therapy and collaborative system approach to amelioration“ [78]; „PIVIP - Programa de intervención para victimas de interferencias parentales“ [139]; „Reunification planning and therapy“ [133]; „Working with alienated children and families - A clinical guidebook“ [11]; „The application of structural family therapy to the treatment of Parental Alienation Syndrome“ [79]; „Understanding and working with the alienated child“ [156]; „Family reflections: A promising therapeutic program designed to treat severely alienated children and their family system“ [125]; „An attachment-based model of Parental Alienation - Foundations“ [40]; „Restoring family connections“ [7]. Letzteres ist eine Methode, die zugelassenen „Mental health professionals“/Therapeuten zur Verfügung steht, die mit betroffenen Eltern und ihren erwachsenen entfremdeten Kindern ambulant arbeiten.

Diese psycho-edukativen und familientherapeutischen Programme, versuchen, schwer entfremdeten Scheidungskindern ihre verlorene Beziehung zu einem Elternteil und ihre verlorene Identität wieder aufzubauen und sie zeigen, dass bei Eltern-KindEntfremdung in Hochkonfliktfällen - entgegen der verbreiteten Meinung - durchaus etwas getan werden kann [151, 152].

Ziele dieser Programme sind die Kontakt- und Beziehungsanbahnung zwischen Kind und ausgegrenztem Elternteil, psycho-edukative Schulung der Eltern, kind-orientierte Elternarbeit, Herstellung von Realität, Korrektur verzerrter Selbst- und Fremdwahrnehmung bei Kind und Eltern, Entlastung des Kindes und Hilfe zur Distanzierung im Loyalitätskonflikt zwischen den Eltern, Wiederaufbau der zerstörten Gefühlsbeziehung durch neue, zukunftsgerichtete gemeinsame Erfahrungen in einem klar strukturierten, sicheren und entspannten Rahmen, Wiederherstellung einer funktionierenden Kommunikation, Verbesserung des Umgangs mit Konflikten und Reorganisation der Familienbeziehungen. Die Kinder lernen, eine realistischere und ausgewogenere Sichtweise ihrer beiden Eltern zu entwickeln und Schwarz-Weiß-Einstellungen abzubauen.

\section{Einige empirische Studien}

1. Die Forscher S. S. Clawar und P. V. Rivlin [42] berichteten aus der bekannten Studie „Children Held Hostage: Dealing with Programmed and Brainwashed Children" (American Bar Association, Section of Family Law, Chicago, 1991) „Wir fanden in unserer Studie, dass Aufenthaltswechsel und erweiterter Kontakt mit dem abgelehnten Elternteil die erfolgversprechendsten Wege sind, um ein Kind zu, deprogrammieren: Je kontinuierlicher und regelmäßiger das Kind Kontakt mit dem entfremdenden Elternteil hat, desto wahrscheinlicher ist es, dass der Entfremdungsprozess sich fortsetzt und der Schaden des Kindes sich verfestigt. “(S. 148ff.) ... . . „Es ist unsere Einschätzung, dass eine der effektivsten Möglichkeiten des Gerichtes die Ankündigung bzw. Realisierung von Sorgerechtswechsel ist. Von den annähernd 400 Fällen, die wir gesehen haben, in denen die Gerichte den Kontakt mit dem abgelehnten Elternteil vermehrt angeordnet haben (und in der Hälfte dieser Fälle gegen die Einwände der Kinder), zeigte sich eine positive Änderung zwischen dem Kind und diesem Elternteil in $90 \%$ dieser Fälle - ein- 
schließlich der Beseitigung oder Reduktion vieler sozial-psychologischer, erzieherischer und körperlicher Probleme, die das Kind vor dieser Maßnahme zeigte." (S. 150) ... ... „Wir hatten die Gelegenheit, Hunderte von Kindern nach einem erfolgten Sorgerechtswechsel $z u$ interviewen und wir können ein Kind für viele andere zitieren: ,Ich hätte niemals von alleine den Kontakt zu meiner Mutter aufgenommen, wenn das Gericht es nicht angeordnet hätte. Nun, da ich es getan habe, habe ich meine Mutter besser kennen gelernt. Sie ist besser, als ich es jemals geglaubt hätte und ich realisiere, dass ich hätte aufwachsen können, ohne sie und ihre Einstellungen zu kennen. Es war wirklich wichtig, - und ich möchte mich dafür bedanken. Auch habe ich gelernt, dass ich nicht alles weiß und dass ich wirklich in Zukunft vorsichtig sein muss, mit einer festgelegten Meinung über eine andere Person. “' (S. 151)

In einer erweiterten Studie von Clawar und Rivlin von 2013 mit 1000 Familien „Children Held Hostage Identifying Brainwashed Children, Presenting a Case, and Crafting Solutions" [43] zeigten sich ähnliche Ergebnisse.

2. Gardner, R. A. [71]: Should courts order PAS-children to visit/reside with the alienated parent? A Follow-up Study. American Journal of Forensic Psychology 19 (3) 61-106 In der vorliegenden Verlaufsstudie beschreibt der amerikanische Kinderpsychiater Richard A. Gardner 99 Entfremdungsfälle, bei denen er als Gutachter unmittelbar involviert war. In diesem Zusammenhang kam er zu dem Schluss, dass das Gericht den Umgang mit dem entfremdeten Elternteil oder den Hauptwohnsitz des Kindes bei diesem anordnen sollte. Die Ergebnisse in den Fällen, in denen diese Anordnungen durchgeführt wurden (22), werden mit den Fällen verglichen, in denen diese Empfehlung nicht entsprochen wurde (77): „Das Gericht entschied sich bei 22 Kindern dafür, entweder den Umgang mit dem entfremdenden Elternteil (eET) einzuschränken oder eine Sorgerechtsänderung vorzunehmen. In allen 22 Fällen trat eine erhebliche Besserung der Ablehnungshaltung ein oder diese verschwand vollständig. ... In 77 Fällen entschied sich das Gericht dafür, keine Sorgerechtsänderung oder beschränkte Kontaktregelung mit eET auszusprechen. Unter diesen Umständen trat bei 70 Fällen (90,9\%) die Entfremdungssymptomatik verstärkt auf. Nur in 7 Fällen $(9,1 \%)$, bei denen keine Sorgerechtsänderung vorgenommen wurde, konnte eine Besserung verzeichnet werden. Es ist also festzustellen, dass Sorgerechtsänderungen und/oder eingeschränkter Kontakt mit eET mit der Reduzierung der Entfremdungssymptomatik in direktem Zusammenhang steht.“

3. In der Kopetski-Follow-up-Studie „The Spectrum of Parental Alienation Syndrome (Part III)“: L. Kopetski, D. C. Rand und R. Rand [103] American Journal of Forensic Psychology 23 (1): 15-43 waren 84 von 423 Familien, die sie von 1975-1990 untersuch- te, PA(S)-Fälle; in 49 Fällen schien die Entfremdung unterbrochen, bei 15 Fällen war sie vollständig ausgeprägt, 8 Fälle waren noch bei Gericht anhängig und 12 mit unbekanntem Ausgang. In 18 der 49 unterbrochenen Entfremdungsfälle hatte das Gericht entweder einen Sorgerechtswechsel zum entfremdeten Elternteil vorgenommen oder den Umgang mit diesem Elternteil angeordnet. In den übrigen „unterbrochenen“ Fällen erreichten die Eltern eine Übereinkunft über Sorgerecht und Umgang. In den Fällen, in denen die Entfremdung „vollständig ausgeprägt“ war, hatte der entfremdende Elternteil das alleinige Sorgerecht bekommen und setzte die gerichtliche Auseinandersetzung so lange fort, bis der Umgang mit dem anderen Elternteil schließlich beendet wurde. Üblicherweise war ein Therapeut mitbeteiligt, der den Entfremdungsprozess unterstützte und der sich auf die Prämisse berief, dass es für das Kind schädlich sei, wenn es aus einer pathologischen symbiotischen Beziehung mit dem entfremdenden Elternteil getrennt würde.

Die Ergebnisse der Studie legen nahe, dass Empfehlungen von Sachverständigen für Sorgerechts- und Umgangsfragen unterscheiden können zwischen „unterbrochener“ und „vollständig ausgeprägter“ Entfremdung bei PAS-Fällen, die sich zum hochgradigen Ende des Spektrums hinbewegen. Die gerichtliche Entscheidung in Bezug auf Sorgerecht und Umgang war wesentlich, wenn es darum ging, die Entfremdung zu unterbrechen oder ihr vorzubeugen. Traditionelle Therapie als primäre Intervention zur Unterbrechung der Entfremdung war ineffektiv, manchmal verschlimmerte sie die Situation noch. Immer mehr Daten weisen darauf hin, dass mittleres bis hochgradiges PA(S) strukturelle Interventionen in Form von gerichtlichen Entscheidungen in Bezug auf Sorgerecht und Umgang erforderlich machen, um den Zugang des Kindes zu beiden Eltern zu schützen. Sorgerechtsentscheidungen, die auf herkömmlichen Konzepten beruhen, wie „Theorie des primären Elternteils“ und „der primären Bindungsbeziehung des Kindes“ gehen davon aus, dass nur ein Elternteil wirklich wichtig für das Kind ist, und dass dessen geäußerte Präferenz unabhängig vom Einfluss eines Elternteils ist. Diese Annahmen vertragen sich nicht mit der Vorstellung, dass Scheidungskinder ebenso wie Kinder in intakten Familien beide Eltern brauchen.

4. Reay, K. M. [125], Family Reflections: A promising therapeutic program designed to treat Severely Alienated children and their family system, American Journal of Family Therapy 43 (2): 197-207 Parental Alienation ist eine Form von psychologischem Kindesmissbrauch. Traditionelle therapeutische Verfahren scheitern bei solchen Fällen von hochgradiger Eltern-Kind-Entfremdung (severe Parental Alienation). Es gibt mindestens zehn wesentliche Gründe dafür, warum bei dieser spezifischen Population traditionelle therapeutische Methoden nicht funktionie- 
ren. Das Family Reflections Reunification Programm (FRRP-Programm) wurde 2012 in einer Pilotstudie mit 22 Kindern, zwischen 8 und 18 Jahren, in 12 Familien durchgeführt. Eine Evaluation mit den Messzeitpunkten direkt nach dem 4-Tages-Programm, nach 3, 6, 9 und 12 Monaten ergab eine $95 \%$ Erfolgsrate bei der Wiederherstellung und Aufrechterhaltung der Beziehung zwischen den Kindern und ihrem einst abgelehnten Elternteil.

Es zeigte sich, dass Kinder - sobald sie sicher und verlässlich aus dem Einflussbereich des entfremdenden Elternteils herausgenommen waren - sich sehr bald wieder emotional mit dem ehemals zurückgewiesenen Elternteil verbanden. Spezifische gerichtliche Anordnungen sind notwendig, um in das FRRP-Programm aufgenommen zu werden. Nach dem erfolgreich verlaufenen Pilotprogramm 2012 wurde FRRP ab Frühjahr 2013 in Kanada und teilweise in USA eingesetzt.

\section{Warshak, R. A. [153], Family Bridges outcome} study. Manuscript in preparation Das Sample besteht aus 83 Kindern (40 Jungen, 43 Mädchen), die an 52 Family Bridges-Workshops teilgenommen haben. Die beiden Hauptziele des Programms sind: die Vorbereitung der Kinder auf das Leben mit dem sorgeberechtigten Elternteil wie es die Aufenthaltsanordnung des Gerichtes vorsieht sowie die Verbesserung der Haltung der Kinder und ihres Verhaltens gegenüber dem abgelehnten Elternteil. Fortschritte in Richtung dieser Ziele wurden durch die Einschätzungen der Eltern und der Workshop-Leiter beurteilt. Verglichen mit $83 \%$ der Kinder, die sich vor dem Workshop dem Kontakt widersetzten, waren es nach dem Workshop nach Einschätzung der Eltern nur noch $6 \%$ und nach Einschätzung der Workshop-Leiter nur noch $4 \%$. Folglich können Gerichte sehen, dass mit der Unterstützung eines Family Bridges Workshops die Chancen hoch sind, dass schwer entfremdete Kinder und Jugendliche, die damit drohen, sich Gerichtsanordnungen zu widersetzen, kooperieren werden.

Alle Elternteile - außer einem - und alle Workshopleiter berichteten von Verbesserungen in der ElternKind-Beziehung, 50\% von ihnen stuften die Beziehung auch dann als „viel besser" ein, wenn der Workshop die negative Einstellung des Kindes nicht lindern konnte und das Kind nicht kooperativ war, mit dem Elternteil zu leben. Auch wenn 7\% der Kinder die Beziehung nach dem Workshop als verschlechtert einstuften, gaben $74 \%$ der Kinder Verbesserung an, 50\% gaben an, die ElternKind-Beziehung sei „etwas besser“. Die Einschätzungen der Beobachter lagen näher bei denen der Eltern, als bei den Kindereinschätzungen: 94\% der Workshopleiter stellten eine „Verbesserung“ fest. $50 \%$ von ihnen gaben „viel besser" an. Daraus folgt: Eltern, Workshopleiter und $75 \%$ der Kinder stimmen darin überein, dass die Eltern-Kind-Beziehung sich vom Anfang bis zum Ende des Workshops verbessert hat.
Verwendet man ein reliables und valides Messinstrument, dann wird die Mehrheit der Kinder, die von Eltern und Workshopleitern zu Beginn des Workshops als schwer entfremdet eingestuft wurden, am Ende der Behandlung von Eltern und Workshopleitern als „viel besser“ bewertet. Der Effekt war statistisch signifikant $(p<0,0001)$ und die Effektstärke, gemessen mit dem Effektmaß Cohens d, war groß. Eltern gaben dem Workshop sehr hohe Bewertungen bei der Erreichung der erklärten Ziele, einschließlich ElternKind-Kommunikation, Konfliktmanagement und die Fähigkeit, miteinander zurecht zu kommen. Die Bewertungen der Kinder waren vorhersehbar niedriger als die der Eltern, aber bezüglich aller Ziele auf dem positiven Ende der Skala. Die Kinder empfanden den Workshop als hilfreich zur Verbesserung der Kommunikation und des Konfliktmanagements. Die durchschnittliche Bewertung des Workshops durch die Kinder war positiv. Mit Hilfe des Workshops sind sie besser in der Lage mit dem Elternteil zu leben, den sie vor dem Workshop abgelehnt haben. Zwei Drittel der Kinder und alle Eltern bewerteten den Workshop als entweder gut oder ausgezeichnet.

\section{Family Separation Clinic, London (K. u. N. Woo-} dall) Die Zahlen der Evaluationsergebnisse lagen bei Einreichung des Artikels noch nicht endgültig vor. Mir wurde aber mitgeteilt, dass vergleichbare Ergebnisse wie bei den Punkten 1.-5. sich andeuten.

\section{Zwei Beispiele aus der Praxis}

A Nach einer hoch konflikthaften Diskussion zwischen Mutter und Schwiegermutter - und verstärkt dann zwei Jahre später, nachdem die Mutter sich von ihrem Mann getrennt und das gemeinsame Haus verlassen hatte -, waren der Kindesvater und die Großmutter väterlicherseits auf die Vorstellung fixiert, die Kindesmutter leide an einer Psychose. Sie konnten ihre Haltung nicht korrigieren, obwohl eine Psychose durch einen vom Gericht beauftragten und auch durch einen zweiten - von der Patientin privat konsultierten - Klinikpsychiater ausgeschlossen worden war und obwohl vom Gericht eine hohe Geldstrafe bei Zuwiderhandlung angedroht worden war. Der Kindesvater, der laut Gerichtsakte als autoritär, unkooperativ, uneinsichtig und manipulativ geschildert wird, äußert gegenüber den Kindern, als auch gegenüber Fachleuten wie Lehrern, Sozialarbeitern, Ärzten und einem Verfahrenspfleger, dass seine Frau an einer Psychose erkrankt sei.

Die Psychose ist eine Projektion von Seiten des Vaters und seiner Mutter auf die Kindsmutter aufgrund einer eigenen psychischen Problematik. Da von außen nicht eingegriffen wurde - die bloße Feststellung, dass bei der Mutter keine Psychose vorliegt , ist nicht ausreichend - haben die Kinder, die seit Jahren beim Vater leben, zu ihrem Selbstschutz, aus Angst und Abhängigkeit diese Projektion übernommen und lehnen 
ihre Mutter rigoros ab (ähnlich wie beim StockholmSyndrom).

Die kindlichen Gefühle von Trauer und Schmerz über den Beziehungsverlust zu ihrer Mutter werden unter dem verzerrenden, negativen Einfluss des Vaters als heftige Wut und Aggression gegenüber der Mutter gezeigt. Die Kinder sind nicht mehr fähig, ihre eigenen Verlustgefühle richtig wahrzunehmen und können dadurch Trauer und Schmerz auch nicht verarbeiten.

Nach einem Video-Transskript (gekürzt; die Personen wurden anonymisiert):

Von einer gerichtlich angeordneten Interview-Situation mit zwei hochgradig entfremdeten Mädchen (10 und 13J.), ihrer Mutter und einer Gutachterin.

Kind 1: $\quad$ Mama, wenn ich dir in die Augen schaue, dann erfüllst du mich mit Mitleid, wie man eine so kranke, mit Medikamenten vollgestopfte Kuh noch frei in der Gegend rumlaufen lässt. Unser Staat ... es ist eine Bedrohung für jeden Menschen, aber ich kann es nicht ändern und ich will es ehrlich gesagt auch nicht ändern. Es ist deine Entscheidung! Wenn du früher oder später und so - wie es mir von mehreren Seiten geschildert wurde - mich zusammengeschlagen hast, bist Du eh nicht mehr meine Mama

Kind 1: $\quad$ Ich habe letztens im Fernsehen eine Sendung gesehen und zwar hat da eine Verrückte einen Polizisten verschleppt und die Polizei musste sich in den verrückten Kopf da reinversetzen. Die haben gesagt, dass die Verrückten die Tatsachen so verschieben, dass alles eine logische Reihenfolge, einen logischen Konsens ergibt. Das ist für sie eine logische Welt

Kind 1: $\quad$ Und das habe ich über Jahre bei meiner Mutter erlebt und beobachten müssen, was mir sehr leid tut

Gutachterin: Willst Du damit sagen, dass deine Mama krank ist?

Kind 1: $\quad$ Was mir wirklich sehr leid tut. Aber mir wurde schon mehrmals von verschiedenen Seiten gesagt, ich kann meiner Mutter nie mehr den Rücken zukehren. Mein ganzes Leben lang nicht

Gutachterin: Weil sie krank ist, willst du sagen?

Kind 1: Ja!

Gutachterin: Du sagst, deine Mama ist verrückt. Hast du denn den Eindruck, dass deine Mama krank ist?

Mutter: $\quad$ Ich glaube dir, dass dich sehr viele Personen bestärkt haben, dass die Mama verrückt ist

Kind 1: Wenn das nicht so wäre, dann gehört sie einfach nur eingesperrt, dann gehört sie einfach nur hinter Schloss und Riegel, in Amerika wäre sie dafür auf den Elektrostuhl gesetzt worden
Kind 1: $\quad$ Ich werde jetzt 13 und meine Schwester 10 und langsam können wir nicht mehr einer Gehirnwäsche unterzogen werden. Langsam geht das nicht mehr und langsam, finde ich, reicht es auch! Weil wir jetzt in ein Alter kommen, wo die Zusammenhänge immer deutlicher werden und wenn ich 18 bin, und es so weitergeht ...

Kind 2: $\quad$ Mit einer Verrückten kann man nicht reden!

Mutter: $\quad$ Aber einen Brief kann man ihr schreiben, ein paar Blumen malen, einfach mal zum Geburtstag gratulieren. Wie wäre denn das?

Kind 1: $\quad$ Man sollte eine Verrückte einfach mal in Ruhe lassen

Gutachterin: Ich muss dir jetzt etwas sagen: Ich habe selbst einmal in der Psychiatrie gearbeitet und auch mit den Verrückten kann man reden. Auch Verrückte sind Menschen

Kind 2: $\quad$ Also so eine Verrückte gehört auf den Elektrostuhl!

Gutachterin: Aber, das ist schon heftig, was du sagst! Kind 2: $\quad$ Sie gehört auf den Elektrostuhl!

Die beiden Mädchen, die aufgrund ihres „eigenen Willens“ beim Vater leben, sind schwer indoktriniert und entfremdet. In dem Interview ist $\mathrm{zu}$ erkennen, dass beide Kinder in pathologischer Weise reagieren, nachdem sie seit mehreren Jahren unter diesem Einfluss leben. Es ist beeindruckend, das bizarre Verhalten der Mädchen zu sehen. Es ist geprägt von Abwertung und aggressiver Zurückweisung ihrer Mutter. Sie nehmen keinen Blickkontakt auf, während sie ihr vorwerfen, unter massivem Medikamenteneinfluss zu stehen, sie gewalttätig misshandelt zu haben, sie mit „Telefonterror“ („124 Anrufe am Abend“) zu belästigen. Sie nennen ihre Mutter in fanatischer Weise „eine psychisch kranke Person“, „eine mit Medikamenten vollgestopfte Kuh“, „eine Bedrohung für jeden Menschen“, die für immer verschwinden solle, durch „Tod auf dem Elektrostuhl“.

Wenn man die Mutter und die Gerichtsakte kennt, wird klar, dass die beiden Mädchen eine völlig unrealistische, verzerrte, geradezu wahnhafte, falsche Überzeugung verinnerlicht haben. Die Anschuldigungen entsprechen dem PA(S)-Symptom „geborgte Szenarien“. Auch andere, von Gardner als PA(S)-typisch beschriebene Symptome sind in diesem Interview deutlich zu erkennen: Verunglimpfungskampagne gegen die Kindesmutter, Phänomen des „eigenständigen Denkens“, absurde Rationalisierungen, fehlende Ambivalenz und fehlende Schuldgefühle. Beide Mädchen zeigen in emotionaler und kognitiver Hinsicht sowie in ihrem Verhalten eine tatsächliche „krankhafte Störung“ als Konsequenz der seit mehreren Jahren anhaltenden suggestiven, realitätsverzerren- 
den Beeinflussung und Indoktrination im väterlichen Umfeld.

Die Gerichtssachverständige hatte PA(S) damals nicht erkannt bzw. negiert und dem Gericht empfohlen, den Umgang auszusetzen, mit der Folge, dass die Mutter ihre beiden Töchter, die inzwischen studieren, seit mehr als 10 Jahren nicht mehr gesehen hat. Die Großmutter (m.s.) ist inzwischen verstorben, ohne ihre Enkelinnen nochmals gesehen zu haben. Der Großvater (m.s.) ist ebenfalls ohne Kontakt und dadurch - bis heute - tief verletzt.

B „Ein Überblick über meine Geschichte“ (von einem ehemals entfremdeten erwachsenen Scheidungskind)

Meine Eltern trennten sich als ich vier Jahre alt war, meine ältere Schwester sechs Jahre. Als ich fünf war, holte uns unser Vater ab - drei Wochen Sommerferien bei ihm und seiner neuen Freundin. Er brachte uns nach den Ferien nicht mehr zurück nach Hause und wir wohnten fortan bei ihm und zogen in ein großes Haus um.

An Vieles aus dieser Zeit kann ich mich nicht mehr erinnern. Es ist als würden mir 4 Jahre meines Lebens fehlen. Ich erinnere mich nur an Bruchstücke oder bestimmte Situationen. Ich kann mich nicht an meine Gefühle aus dieser Zeit erinnern. Auch nicht daran, dass ich meine Mutter gehasst oder vermisst und manchmal an sie gedacht hätte. Für mich ist es, als hätte sie einfach vier Jahre lang nicht existiert.

Über die gesamte Zeit verteilt fanden immer wieder psychologische Gutachten statt und es sollte Treffen mit dem Jugendamt und unserer Mutter geben, die allerdings selten stattfanden. Das Jugendamt war leider nicht in der Lage sich durchzusetzen. Ein Treffen ohne meinen Vater und meine damalige Stiefmutter war nicht möglich. Das Jugendamt beschloss, dass es keinen Sinn macht, es weiter zu versuchen.

Als ich neun Jahre alt war, wurden meine Schwester und ich am letzten Schultag vor den Sommerferien von zwei Polizisten und der Anwältin meiner Mutter von der Schule abgeholt und in ein Heim gebracht. Die ersten Wochen waren furchtbar. Ich weinte jede Nacht. Nach einiger Zeit fing es an mir zu gefallen. Meine Mutter besuchte uns immer wieder gemeinsam mit einer Dame vom Jugendamt. Bei den ersten Treffen konnte ich nicht auf sie zugehen, dann kamen wir uns allmählich wieder näher. Wir verbrachten auch Zeit mit ihr alleine. Irgendwann zog unsere Mutter mit uns in eine Dachwohnung des Heimes. Dort lebten wir für ein paar Monate und besuchten dort die Schule. Wir gingen auch mal am Wochenende mit ihr nach Hause oder bekamen Besuch und machten Ausflüge mit unseren Stiefbrüdern und unserem Stiefvater. Unser Vater und unsere Stiefmutter durften uns nicht besuchen. Kurz vor Weihnachten durften wir mit unserer Mutter nach Hause. Wir lebten dann gemeinsam in einem Haus mit ihr, unserem Stiefvater und unseren Stiefbrüdern. Wir durften unseren Vater nur in Begleitung einer Heimmitarbeiterin besuchen. Als wir fort waren, verließ meine Stiefmutter meinen Vater. Sie nahm alle Wertsachen mit, räumte sogar unsere Sparbücher leer und verschwand. Seither besuchten wir meinen Vater auch immer wieder an Wochenenden oder in den Ferien. Meine Schwester lehnte das irgendwann ab. Ich ging weiterhin $\mathrm{zu}$ ihm.

Als ich fünfzehn Jahre alt war kam plötzlich alles wieder hoch, vor allem in Träumen. Ich fing an, mich selbst $\mathrm{zu}$ therapieren und kam damit ziemlich weit. Ich hatte damals panische Angst vor Polizisten und war klaustrophobisch. Ich fühlte mich innerlich komplett leer und zerrissen. Ich ritzte mich, um aus Panikattacken wieder herauszukommen. Ich hatte mich sehr zurückgezogen von Freunden und Familie.

Mein Appell an Fachkräfte: Greift ein! Die Macken, die ich vom Eingreifen der Justiz und des Jugendamts davontrug, konnte ich bis heute soweit bearbeiten, dass sie nicht mehr existieren oder mich nur minimal oder selten beeinflussen. Was mich bis heute innerlich zerreißt, ist das Fehlen so vieler Erinnerungen, der Verlust der gemeinsamen Zeit und vor allem, was ich meiner Mutter angetan habe. Was wäre wohl passiert oder wer oder wie wäre ich heute, wenn damals nicht diese radikalen Schritte erfolgt wären. Aber ich weiß, dass der Gedanke für mich unerträglich ist, keinen Kontakt mehr zu meiner Mutter zu haben. Wahrscheinlich hätte ich sie verstoßen, sie wäre kein Teil meines Lebens mehr gewesen. Ich war schon immer ein „Mama-Kind“. Dass ich sie nicht in meinem Leben hätte haben wollen, ist einfach grundlegend falsch. Es hätte mich irgendwann zerstört, ohne sie zu sein. Dank der Herausnahme und Wiederzusammenführung habe ich heute eine gute Beziehung zu beiden Elternteilen.

Ich habe von vielen Fällen gehört und gelesen, in denen die Wichtigkeit von externer Hilfe (durch Jugendamt und gerichtliche Regelungen) von entfremdenden Eltern, als auch von Fachkräften nicht realisiert wird (dazu gehört auch die ignorante Ablehnung der Existenz von „Parental Alienation“). Ebenso wie die Notwendigkeit der Isolierung vom entfremdenden Elternteil und der Zusammenführung mit dem entfremdeten. Das macht mich traurig und wütend. Ich halte es für sehr wichtig einzugreifen und den entfremdeten Kindern zu helfen. Und das möglichst früh, sodass die psychischen Folgen halbwegs gering bleiben. Ein Kind braucht beide Eltern oder zumindest die Möglichkeit, von beiden Eltern ein gutes Bild und regelmäßigen Kontakt mit ihnen zu haben. Unter Manipulation ist das nicht möglich. Ich nannte es bereits „radikale Lösung“ - weshalb es offenbar nicht so gemacht wird. „Das kann man doch den Kindern nicht antun." Aber wenn alle anderen Versuche der Zusammenführung missglückt sind, ist es die einzige Möglichkeit! Wenn ich mich an das Heim erinnere, war es eine gute Zeit. Ich hatte in der Schule gute Freunde gefunden, fühlte mich in der Stadt wohl, mochte was das Heim zu bieten hatte (Schwimmbad, Tobe- 
Raum etc.), schätzte die Betreuerinnen und Psychologen. Rückblickend war es das letzte Mal, dass ich wirklich unbeschwert glücklich war. Alle Folgen, die dieses „massive Eingreifen“ hatte, konnte ich letztlich erfolgreich bearbeiten. Das was bis heute an mir nagt, ist, dass mir vier Jahre meines Lebens genommen wurden und ich Dinge tat, die ich meiner Mutter niemals hätte antun dürfen. Das belastet mich bis heute und ich werde es wohl nie schaffen, das ausreichend zu verarbeiten. Es scheint mir definitiv das kleinere Übel, solch manipulierte und entfremdete Kinder aus der Familie herauszuholen, als den Entfremdungsprozess jahrelang laufen zu lassen.

\section{Stuttgart, den 28.09.2015 - D.}

\section{Abschlussbemerkungen}

In Anbetracht der exemplarisch in den praktischen Beispielen A und B geschilderten tragischen Erfahrungen und der psychotraumatischen Langzeitfolgen von pathologischer Entfremdung und Kontaktverlust können Entfremdungsprozesse für Kinder und Eltern nicht als familiäre Privatangelegenheit angesehen werden. Im Falle von hoch konflikthaften Trennungs-/Scheidungsstreitigkeiten, bei denen die Gefahr einer PA(S)-Entwicklung durch massive Instrumentalisierung der Kinder besteht, ist eine frühzeitige präventive, interdisziplinäre Zusammenarbeit aller beteiligten Berufsgruppen erforderlich, um den Elternkonflikt zu reduzieren und den „Point of no return“ in der Gefährdung einer Eltern-Kind-Beziehung zu verhindern.

Wo das aufgrund der psychopathologischen Situation eines oder beider Elternteile nicht möglich ist, sind psychologische Pflichtberatungen und direktive oder konfrontative Interventionen und/oder strukturelle familiengerichtliche Maßnahmen erforderlich (wie Sanktionen oder ein Sorgerechtstransfer unter psychologischer Begleitung), denn die Grenzen der familialen Autonomie sind hier erreicht. Parental Alienation ist dann keine Frage des Sorgerechts mehr, sondern eine Frage des Kinderschutzes. Erfahrungsberichte aus Kanada, England, USA, Australien, Spanien, vereinzelt auch aus Deutschland, ermutigen zu einem stärker direktiven und konfrontativen Vorgehen. Sie werden gestützt durch Forschungsergebnisse, die die Wirksamkeit von Interventionsprogrammen bestätigen. Gegebenenfalls muss über ergänzende gesetzliche Regelungen nachgedacht werden.

Was die künftige wissenschaftliche Forschung (vor allem in den Bereichen Entwicklungspsychologie, Kinder-/Erwachsenenpsychiatrie und Psychosomatik) angeht, sind weitere systematische, empirische Studien mit größeren Stichproben, standardisierten Maßstäben und geeigneten wissenschaftlichen Methoden nötig. Dadurch sollten die noch bestehenden Kontroversen über Gültigkeit und Reliabilität der Diagnose PA(S) - im Sinne einer kindlichen Beziehungsstörung - ausgelöst durch schwer manipulatives Fehlverhal- ten von Eltern und/oder anderen wichtigen Bezugspersonen - beendet werden. Die wissenschaftliche Validität des PA(S)-Konzeptes ist empirisch weiter zu untermauern. Dazu gehören auch weitere epidemiologische klinische Untersuchungen in Bezug auf Langzeitverlauf/-folgen von PA(S) und auf Wirksamkeit effektiver Interventionen bei den verschiedenen Schweregraden der Störung.

Die Resultate künftiger Studien sollten helfen, unser Verständnis darüber weiter zu verbessern, wie bei steigenden Scheidungszahlen pathologisch entfremdeten Kindern und ihren Familien besser geholfen werden kann. - Und was die Aufnahme der Diagnose „Parental Alienation“ in die neue „International Classification of Diseases“ (ICD 11) der Weltgesundheitsorganisation (WHO) angeht, darf man letztlich gespannt sein, wie sich die zuständigen Fachleute entscheiden werden.

Interessenkonflikt W. von Boch-Galhau gibt an, dass kein Interessenkonflikt besteht.

Open Access Dieser Artikel wird unter der Creative Commons Namensnennung 4.0 International Lizenz (http:// creativecommons.org/licenses/by/4.0/deed.de) veröffentlicht, welche die Nutzung, Vervielfältigung, Bearbeitung, Verbreitung und Wiedergabe in jeglichem Medium und Format erlaubt, sofern Sie den/die ursprünglichen Autor(en) und die Quelle ordnungsgemäß nennen, einen Link zur Creative Commons Lizenz beifügen und angeben, ob Änderungen vorgenommen wurden.

\section{Literatur}

1. Ackermann MJ, GouldJW. Child custodyand access. In:Cutler BL, Zapf PA, Hrsg. Individual and situational influences in criminaland civil contexts. APAhandbookofforensic psychology, vol. 1. Washington D.C.: American Psychological Association; 2015. S. 454-7.

2. Andritzky W. Verhaltensmuster und Persönlichkeitsstruktur entfremdender Eltern: Psychosoziale Diagnostik und Orientierungskriterien für Interventionen. Psychother Psychiatr Psychother Med Klin Psychol. 2002a;7(2):166-82.

3. Andritzky W. Zur Problematik kinderärztlicher Atteste bei Umgangs- und Sorgerechtsstreitigkeiten. Kinder Jugendarzt.2002b;33(11):885-9. und 2002b; 33 (12):984-990.

4. Baker AJL. The long-term effects of parental alienation on adult children: a qualitative research study. Am J Fam Ther. 2005a;33(4):289-302.

5. Baker AJL. The cult of parenthood: a qualitative study of parental alienation. Cultic Stud Rev. 2005b;4(1):1-29.

6. Baker AJL. Children of parental alienation syndrome breaking the ties that bind. New York, London:W.W. Norton \&Company; 2007.

7. Baker AJL. Restoring family connections. 2016. www. amyjlbaker.wix.com/rfcprogram. Zugegriffen: 14. Dez. 2016.

8. Baker AJL, Darnall D. Behaviors and strategies employed in parental alienation: a survey of parental experiences. JDivorce Remarriage. 2006;45(1/2):97-123.

9. Baker AJL, Darnall D. A construct study of the eight symptoms of severe parental alienation syndrome: a survey of parental experiences. J Divorce Remarriage. 2007;47(1/2):55-75. 
10. BakerAJL,BurkhardB,KellyJ.Differentiatingalienatedfrom not alienated children: a pilot study. J Divorce Remarriage. 2012;53(3):178-93.

11. Baker AJL, Sauber SR, Hrsg. Working with alienated children and families - a clinical guidebook. New York: Routledge; 2013.

12. Baker AJL, Kase-Gottlieb L, Verrocchio MC. The reality of parentalalienation: commentaryon ,Judicial decision-making in familylaw proceedings byClemente, Padilla-Racero, Gandoy-Crego, Reig-Botella \& Gonzalez-Rodriquez“. Am J Fam Ther. 2016;44(1):46-51.

13. Bäuerle S, Moll-Strobel H, Hrsg. Eltern sägen ihr Kind entzwei, Trennungserfahrungen und Entfremdung von einem Elternteil. Donauwörth:Auer; 2001.

14. Beermann M. Ein Prozess wird zum Justiz-Skandal. Süddeutsche Zeitung Nr. 121 vom 26.05.2004 (Themen des Tages)

15. Bensussan P. Alienation parentale, abus psychologique de l'enfant et DSM-5. Encephale. 2017;43:510-5.

16. Bernet W. False statements and the differential diagnosis of abuse allegations. J Am Acad Child Adolesc Psychiatry. 1993;32(5):903-10.

17. Bernet W. Sexual abuse allegations in the context of child custody disputes. In: Gardner RA, Sauber RS, Lorandos D, Hrsg. Conceptual, clinical and legal considerations. Handbook of parental alienation syndrome. Springfield: C. C. Thomas; 2006. S. 242-63.

18. Bernet W. Parental alienation disorder and DSM-V. Am J Fam Ther. 2008;36(5):349-66.

19. Bernet W. Parental alienation, DSM-5 and ICD-11. Springfield:C.C. Thomas; 2010.

20. BernetW. Parentalalienation-misinformation versus facts. Judges J.2015;54(3):23-7.

21. Bernet W, Baker AJL. Parental alienation, DSM-5, and ICD-11: response to critics. J Am Acad Psychiatry Law. 2013;41(1):98-104.

22. Bernet W, von Boch-Galhau W, Baker AJL, Morrison S. Parental alienation, DSM-5 and ICD-11. Am J Fam Ther. 2010;38(2):76-187.

23. Bernet W, Baker AJL, Verrocchio MC. Symptom checklist90-revised scores in adult children exposed to alienating behaviors: an Italian sample.J Forensic Sci. 2015;60(2):357-62.

24. Bernet W, Wamboldt MZ, Narrow WE. Child affected by parental relationship distress. J Am Acad Child Adolesc Psychiatry. 2016a;55(7):571-9.

25. Bernet W, Öngider-Gregory N, Reay K, Rohner RP. An objective measure of splitting in parental alienation: the parental acceptance-rejection-questionnaire. J Forensic Sci. 2016b;55(10):S201. Supplement.

26. BlankM.Anmerkungen zur Persönlichkeitsstruktur des betreuenden Elternteils als mögliche zentrale Ursache für die Entstehung eines elterlichen Entfremdungssyndroms. In: vonBoch-GalhauW,KodjoeU,AndritzkyW,KoeppelP,Hrsg. Das Parental Alienation Syndrom - Eine interdisziplinäre Herausforderung für scheidungsbegleitende Berufe/The Parental Alienation Syndrome (PAS) - An Interdisciplinary Challenge for Professionals Involved with Divorce. [Deutsch/Englisch]. Berlin: VWB-Verlag Wissenschaft und Bildung; 2003. S. 343-51.

27. von Boch-Galhau W. Parental alienation and parental alienation syndrome/disorder-a serious form of psychological child abuse - with case examples. Berlin: VWB-Verlag Wissenschaft und Bildung; 2013a.

28. von Boch-Galhau W. Kritische Anmerkungen zu J M Fegert: „Endgültiges Aus für das Parental Alienation Syndrome im amerikanischen Klassifikationssystem DSM-5“ (ZKJ2013b, S. 190 f.).ZKindschaftsrecht Jugendhilfe. 2013b;10:400.
29. von Boch-Galhau W, Kodjoe U. Parental Alienation Syndrome - Psychische Folgen für erwachsene Scheidungskinder und für betroffene Eltern. Interdiszip Fachz KindesmisshandlVernachläss. 2003;6(1/2):66-97.

30. BowlbyJ.Attachment.Attachmentandloss, Bd. 1. NewYork: Basic Books; 1969.

31. Bowlby J. Separation. Attachment and loss, Bd. 2. New York: Basic Books; 1973.

32. Bricklin B, Elliott G. Psychological test-assisted detection of parental alienation syndrome. In: Gardner RA, Sauber SR, Lorandos D, Hrsg. Conceptual, clinical and legal considerations. The international handbook of parental alienation syndrome. Springfield: Thomas; 2006. S. 264-75.

33. Broca R. Un changement de paradigme: le modèle de Cochem. In: Broca R, Odinetz O, Hrsg. Séparations conflictuelles etaliénation parentale-Enfants en danger. Lyon:Ed. Chronique Sociale; 2016. S.317-25.

34. Broca R, Odinetz O, Hrsg. Séparations conflictuelles et aliénation parentale-Enfants en danger. Lyon: Ed. Chronique Sociale; 2016.

35. Brockhausen T. Parental alienation and the new Brazilian law, chap. 14. In: Lorandos D, Bernet W, Sauber SR, Hrsg. Parental alienation - the handbookfor mental health andlegal professionals. Springfield: C. C. Thomas;2013. S. 463-84.

36. Bron B, Strack M, Rudolph G. Childhood experiences of loss and suicide attempts: significance in depressive states of major depressed and dysthymic or adjustment disordered patients. JAffect Disord. 1991;23:165-72.

37. Bruch CS. Parental alienation syndrome: getting it wrong in child custody cases. Fam Law Q. 2001;35(3):527-52.

38. Burrill J. Descriptive statistics of the mild, moderate, and severe characteristics of parental alienation syndrome. In: Gardner RA, Sauber SR, Lorandos D, Hrsg. Conceptual, clinical and legal consideration. The international handbook of parental alienation syndrome. Springfield: C. C. Thomas; 2006. S. 49-55.

39. Ceci SJ, Bruck M. Jeopardy in the courtroom - A scientific analysis of children's testimony. 6. Aufl. Washington, D.C.: American Psychological Association; 2012.

40. Childress C. An attachment-based model of parental alienation-foundations. Claremont: Oaksong Press; 2015.

41. Cierpka M, Cierpka A. Die Identifikationen eines missbrauchten Kindes. Psychotherapeut. 2000;42:98-105.

42. Clawar SS, Rivlin BV. Children held hostage. Dealing with programmed and brainwashed children. Chicago: American Bar Association, Division of Family Law; 1991.

43. Clawar SS, Rivlin BV. Children held hostage, identifying brainwashed children, presenting a case, and crafting solutions. Chicago: American Bar Association, Division of Family Law; 2013.

44. Clemente M, Padilla-Racero D. Are children susceptible to manipulation? Child Youth Serv Rev. 2015a;51:101-7.

45. Clemente M, Padilla-Racero D, Gandoy-Crego M, ReigBotella A, Gonzalez-Rodriguez R. Judicial decision-making in family law proceedings. Am J Fam Ther. 2015b;43:314-25.

46. Clemente M, Padilla-Racero D. When courts accept what science rejects: custody issues concerning the alleged "parental alienation syndrome". J Child Custody. 2016;13(2):126-33.

47. Cour de Cassation in France. Le syndrome d'aliénation parentale reconnu par la Cour de cassation: les premiers pas d'une révolution dans le contentieux familial? Urteil Nr. 660 vom 26. Juni 2013 (12-14.392), ECLI:FR:CCASS:2013:C100660. 2013. http://www.courde cassation.fr/jurisprudence_2/premiere_chambre_civile 568/660_26_26933.html.Zugegriffen: 14. Dez. 2016, Nationales Berufungsgericht. 
48. Darnall DC. The psychosocial treatment of parental alienation. Child Adolesc Psychiatr Clin NAm. 2011;20:479-94.

49. Darnall DC. Mild cases of parental alienation. In: Lorandos D, BernetW, SauberSR, Hrsg. Parentalalienation-thehandbook for mental health and legal professionals. Springfield: C.C. Thomas; 2013. S. 74-96.

50. Deegener G, Körner W, Hrsg. Kindesmisshandlung und Vernachlässigung - Ein Handbuch. Göttingen: Hogrefe; 2005.

51. Dossier Special Outreau, www.acalpa.org

52. Dum CT. Parental alienation initiatives around the world, chap. 13.In:Lorandos D, BernetW, SauberSR, Hrsg. Parental alienation - the handbook for mental health and legal professionals. Springfield: C. C. Thomas; 2013a. S. 425-62.

53. Dum CT. Chap. 13, parental alienation initiatives around the world. In: Lorandos B, Sauber, Hrsg. Parental alienation - the handbook for mental health and legal professionals. Springfield:C.C. Thomas;2013b.S.439-44. European Court of Human Rights in Strasbourg

54. Eckhardt-Henn A. Artifizielle Störungen und Münchhausen-Syndrom. In: Kernberg OF, Dulz B, Sachsse U, Hrsg. Handbuch der Borderline-Störungen. Stuttgart: Schattauer;2000.S.331-45.

55. Faller KC. The parental alienation syndrome: what is it and what data supportit? Child Maltreat. 1998;3(2):100-15.

56. Fegert JM. Parental Alienation oder Parental Accusation Syndrome? Die Frage derSuggestibilität, Beeinflussungund Induktion in Umgangsrechtsgutachten. Kindschaftsrechtl Prax. 2001;4(1):3-7. und 2001;4 (2):39-42.

57. Fegert JM. Endgültiges Aus für das Parental Alienation Syndrom (PAS) im amerikanischen Klassifikationssystem DSM-5.ZKindschaftsrecht Jugendhilfe. 2013a;5:190-1.

58. FegertJM. Wasfehlt, sindlediglich quantifizierendeStudien - eine Replik.ZKindschaftsrecht Jugendhilfe.2013b;10:401.

59. Felitti VJ, Anda RF, Nordenberg D, Williamson DF, Spitz AM, Edwards V, Koss MP, Marks JS, et al. Relationship of childhood abuse and household dysfunction to many of the leading causes of death in adults the Adverse Childhood Experiences (ACE) study. Am J Prev Med. 1998;14:245-58.

60. Fernández Cabanillas FJ, Hrsg. Manual del Sindrome de Alienación Parental. Barcelona: Editorial Paidós; 2017.

61. Festinger L. A theory of cognitive dissonance. Stanford: Stanford University Press; 1957.

62. Fidler BJ, Bala N, Birnbaum R, Kavassalis K. Challenging issues in child custody assessments: a guide for legal and mental health professionals. Toronto: Thomson Carswell; 2008.

63. Fidler B, Bala N, Saini MA. Children who resist postseparation parental contact - A differential approach for legal and mental health professionals. American psychology-law society series. Oxford: University-Press; 2013.

64. Figdor H. Psychodynamik bei sogenannten „Entfremdungsprozessen“ im Erleben von Kindern - ein kritischer Beitrag zum PAS-Konzept. In: von Boch-Galhau W, Kodjoe U, Andritzky W, Koeppel P, Hrsg. Das Parental Alienation Syndrom - Eine interdisziplinäre Herausforderung für scheidungsbegleitende Berufe/ The Parental Alienation Syndrome(PAS)-AnInterdisciplinaryChallengefor Professionals Involved with Divorce. [Deutsch/Englisch]. Berlin: VWB-Verlag Wissenschaft und Bildung;2003. S. 187-206.

65. Foran HM, Beach SRH, Smith Slep AM, Heyman RE, Wamboldt MZ, Hrsg. Family problems and family violence reliable assessment and the ICD-11. New York: Springer; 2013.

66. Friedlander S, Walters MG. When a child rejects a parent: tailoring the intervention to fit the problem. Fam Court Rev. 2010;48(1):98-111.
67. Füchsle-Voigt T. Verordnete Kooperation im Familienkonflikt als Prozess der Einstellungsänderung: Theoretische Überlegungen und praktische Umsetzung. Fam Partnersch Recht. 2004;10(11):600-2.

68. Gardner RA. Recent trends in divorce and custody litigation. Acad Forum. 1985;29(2):3-7.

69. Gardner RA. The parental alienation syndrome. 2. Aufl. Cresskill: Creative Therapeutics; 1998.

70. Gardner RA. Therapeutic interventions for children with parental alienation syndrome. Cresskill: NJ; 2001a.

71. Gardner RA. Should courts order PAS-children to visit/ reside with the alienated parent? A Follow-up Study. Am J Forensic Psychol.2001b;19(3):61-106.

72. Gardner RA, Sauber SR, Lorandos D, Hrsg. Conceptual, clinical and legal considerations. The international handbook of parental alienation syndrome. Springfield: C. C. Thomas; 2006.

73. Gebhardt G. Victims of another war - the aftermath of parental alienation. 2005. www.victimsofanotherwar.com. Zugegriffen:21.Febr. 2017.

74. Gebhardt G. Sarah Cecilie. 2015. https://www.youtube. com/watch?v=3qgj3WXYHyo. Zugegriffen: 27. Juni 2017, Original Englische Version mitDeutschen Untertiteln.

75. van Gijseghem H. L'aliénation parentale: les principales controverses. Rev Daction Jurid Soc. 2004;237:11-7.

76. Gödde M. Indikationsspezifische Strategien, - Indikation: Umgangsverweigerung bzw. Kontaktverunsicherung. In: Fthenakis WE, Hrsg. Begleiteter Umgang von Kindern Ein Handbuch für die Praxis. München: C. H. Beck; 2008. S. 245-305.

77. Gordon RM. The Medea complex and the parental alienation syndrome: when mothers damage their daughter's ability to love a man. In: Fenchel GH, Hrsg. The motherdaughter relationship: echoes through time. Northvale: Jason Aronson; 1998. S. 207-25.

78. Gottlieb LJ. Parental alienation syndrome: a family therapy and collaborative systems approach to amelioration. Springfield:C.C. Thomas; 2012.

79. Gottlieb LJ. The application of structural family therapy to the treatment of parental alienation syndrome. In: Baker AJL, Sauber SR, Hrsg. Working with alienated children and families - a clinical guidebook. New York: Routledge; 2013. S. 209-31.

80. Hachenberg, M. Elterliche Entfremdung - Diagnose und mögliche Folgen, Psychologisches Institut, Würzburg: UniversitätWürzburg: Diplomarbeit; 2014

81. Harmann JJ, Biringen Z. Parents acting badly; how institutions and society promote the alienation of children from their loving families. Fort Collins: The Colorado Parental Alienation Project; 2016.

82. Heiliger A, Hack EK, Hrsg. Väter um jeden Preis - zur Kritik am Sorge- und Umgangsrecht. München: Verlag Frauenoffensive; 2008

83. Hellblom-Sjögren L. Making a Parent Dangerous - PAS in Sweden and Norway/Einen Elternteil gefährlich machen PAS in Schweden und Norwegen. In: von Boch-Galhau W, Kodjoe U, Andritzky W, Koeppel P, Hrsg. Das Parental Alienation Syndrom - Eine interdisziplinäre Herausforderung für scheidungsbegleitende Berufe/The Parental Alienation Syndrome (PAS) - An Interdisciplinary Challenge for Professionals Involved with Divorce. [German/English]. Berlin: VWB-Verlag Wissenschaft und Bildung; 2003. S.235-48.

84. Hetherington EM, Kelly J. For better or for worse divorce reconsidered. New York, London:W.W. Norton \& Company. Inc; 2002.

85. Heuchemer MO. Kardinalfehler der Rechtsprechung im Umgangsrecht-Rechtsfehler, Rechtsverletzungen und die 
(in-)Effizienz prozess- und materiell-rechtlicher Korrekturmechanismen im Instanzenzug am Beispiel der ElternKind-Entfremdung (Parental Alienation Syndrome "PAS"). Fam Recht. 2017;7:368-72.

86. Hinterhofer H, Müller T, Payrhuber D, Pletzer R. Das Eltern-Entfremdungs-Syndrom (PAS) aus medizinischer und rechtlicher Sicht. Jurist Bl. 2013;135:420-8 (Teil I). und 2013; 135:488-496 (Teil II).

87. Hirigoyen M-F. L'aliénation parentale comme abus de faiblesse. In: Broca R, Odinetz O, Hrsg. Séparations conflictuelles et aliénation parentale-Enfants en danger. Lyon: Ed. Chronique Sociale;2016. S. 102-15.

88. Hummel K. Entsorgte Väter - Der Kampf um die Kinder: Warum Männer weniger Recht bekommen. Köln: LübbeVerlag; 2010.

89. Johnston JR. Children of divorce who reject a parent and refuse visitation: recent research and social policy implications for the alienated child. Fam Law Q. 2005;38(4):757-75.

90. Johnston JR, Roseby V. In the name of the child: a developmental approach to understanding and helping children of conflicted und violent divorce. New York: Free Press; 1997.

91. JohnstonJR, Girdner LK. FamilyAbductors:Descriptiveprofiles and preventive interventions. Juvenile justice bulletin. Washington, D.C.: U.S. Department of Justice; 2001.S. 1-7.

92. Kelly JB, Johnston JR. The alienated child, a reformulation of parental alienation syndrome. Fam Court Rev. 2001;39(3):249-66.

93. Kelly JB. Commentary on "Family bridges: using insights from social science to reconnect parents and alienated children" (Warshak, 2010). Fam Court Rev. 2010;48(1):81-90.

94. Kernberg OF, Dulz B, Sachsse U, Hrsg. Handbuch der Borderlinestörungen. Stuttgart: Schattauer; 2000.

95. Klenner W. Rituale der Umgangsvereitelung. Z Ges Familienrecht. 1995;42(24):1529-35.

96. Klosinski G. Psychological maltreatment in the context of separation and divorce. Child Abuse Negl. 1993;17:557-63.

97. Kodjoe U, Koeppel P. The Parental Alienation Syndrome (PAS). Amtsvormund. 1998;71(1):9-26. und 1998; 71 (2): 135-140.

98. Kodjoe U. Auswirkungen des Vater-Kind-Kontaktverlustes: der immaterielle Schaden aus psychologischer Sicht - Anmerkungen zur Elsholz-Entscheidung des Europäischen Gerichtshofs für Menschenrechte. Amtsvormund. 2000;73(8):641-3.

99. van der KolkBA, McFarlane AC, Weisaeth L, Hrsg. Traumatic Stress, Grundlagen und Behandlungsansätze - Theorie, Praxis und Forschung zu posttraumatischem Stress sowie Traumatherapie. Paderborn: Junfermann;2000.

100. KopetskiL. Identifying cases of parentalienation syndrome, part I. Colo Lawyer. 1998a;27(2):65-8.

101. KopetskiL.Identifying Cases ofParentAlienation Syndrome -Part II. Colo Lawyer. 1998b;27(3):61-4.

102. Kopetski L. Commentary: parental alienation syndrome. In: Gardner RA, Sauber SR, Lorandos D, Hrsg. Conceptual, clinical and legal considerations. The international handbook of parental alienation syndrome. Springfield: C. C. Thomas; 2006. S.378-90.

103. Kopetski L, Rand D, Rand R. The spectrum of parental alienation syndrome, (part III): the Kopetski follow-up study. Am J Forensic Psychol. 2005;23(1):15-43.

104. Kopetski L, Rand D, Rand R. Incidence, gender, and false allegations of child abuse: data on 84 parental alienation syndrome cases. In: Gardner RA, Sauber SR, Lorandos D, Hrsg. Conceptual, clinical and legal considerations. The international handbook of parental alienation syndrome. Springfield: C. C. Thomas;2006. S. 65-70.
105. Loftus E, Ketcham K. Die therapierte Erinnerung vom Mythos der Verdrängung bei Anklagen wegen sexuellen Missbrauchs. Hamburg: Klein; 1995.

106. Lorandos D. Parental alienation syndrome: detractors and the junk science vacuum. In: Gardner RA, Sauber SR, Lorandos D, Hrsg. Conceptual, clinical and legal considerations. International handbook of parental alienation syndrome. Springfield: C. C. Thomas;2006. S. 397-418.

107. Lorandos D. Parental alienation and North American law, chap. 12, (PA-Urteile in USA and Canada). In: Lorandos D, Bernet W, Sauber SR, Hrsg. Parental alienation - the handbook for mental health and legal professionals. Springfield: C. C. Thomas; 2013.S.348-424.

108. Lorandos D, Sauber SR, Bernet W, Hrsg. Parental alienation: the handbook for mental health and legal professionals. Springfield: C. C. Thomas; 2013.

109. Lowenstein LF. The psychological effects and treatment of the parental alienation syndrome. In: Gardner RA, Sauber SR, Lorandos D, Hrsg. Conceptual, clinical and legal considerations. The international handbook of parental alienation syndrome. Springfield: C. C. Thomas; 2006. S. 292-301.

110. Lowenstein LF. Parental alienation. How to understand and address parental alienation resulting from acrimonious divorce or separation. Dorset: Russelt House; 2007.

111. Marique B. Le modèle de consensus parental de Dinant, Belgique. In: Broca R, Odinetz O, Hrsg. Séparations conflictuelles et aliénation parentale-Enfants en danger. Lyon:Ed. Chronique Sociale; 2016. S. 326-38.

112. MinuchinS. Familles en therapies. Paris: Erès; 1998.

113. Mullen PE, Martin JL, Anderson JC, Romans SE, Herbison GP. The long-term impact of the physical, emotional and sexual abuse of children: a community study. Child Abuse Negl. 1996;20(1):7-21.

114. Oberlandesgericht Frankfurt/M. 6WF168/00, Urteil vom 26. Okt. 2000.ZGes Familienrecht. 2001;48(10):638.

115. Palandt O, Bassenge P, Brudermüller G. Bürgerliches Gesetzbuch. 68. Aufl. München: BeckJuristischerVerlag; 2008.

116. Center for Knowledge, Vanderbilt University, Medical Center.Parentalalienation database.2016. www.mc.vanderbilt. edu/pasg.Zugegriffen:20.Dez. 2016.

117. Paricard S. Le syndrome d'alienation parentale, catalyseur d'un conflit des droits de l'enfant. In: Neinrinck C, Bruggemann M, Hrsg. La Convention Internationale des Droits de l'Enfant, une Convention Particulière. Paris: Dalloz; 2013. S.71-89.

118. Petri H. Das Drama der Vaterentbehrung. 3. Aufl. Freiburg: Herder; 2011.

119. Pope HG, Oliva PS, Hudson JI. The scientific status of research on repressed memories. In: Faigmann DL, Kaye DH, Saks MJ, Sanders J, Hrsg. Modern scientific evidence: the law and science of expert testimony. Vol I. Eagan: West Publishing; 1999. S. 115-55.

120. Rand DC. Munchhausen syndrome by proxy: a complex type of emotional abuse responsible for some false allegations of child abuse in divorce. Issues Child Abuse Accusations. 1993;5(3):135-55.

121. Rand DC. The spectrum of parental alienation syndrome (part I).Am J Forensic Psychol. 1997a;15:23-51.

122. Rand DC. The spectrum of parental alienation syndrome (part II).Am J Forensic Psychol. 1997b;15:39-92.

123. Rand DC. Parental alienation critics and the politics of science. Am J Fam Ther. 2011;39:48-71.

124. Reay K. Toxic divorce: a workbook for alienated parents. Penticton: Routledge, Taylor \& Francis; 2011.

125. ReayK.Familyreflections: a promising therapeutic program designed to treat severely alienated children and their family system. Am J Fam Ther. 2015;43(2):197-207. 
126. Rudolph J. Du bistmein Kind-Die „Cochemer Praxis“-Wege zu einem menschlichen Familienrecht. Berlin: Schwarzkopf \&Schwarzkopf; 2007.

127. Rueda CA. An inter-rater reliability study of parental alienation syndrome.Am J Fam Ther. 2004;32(5):391-403.

128. Saini M, Drozd L, Olesen N. Parenting plan evaluations: applied research for the family court. 2. Aufl. Oxford: Oxford University Press; 2016

129. Salgo L. Das Wohl des Kindes unter den Aspekten gesetzlicher Einflüsse. In: Brisch KH, Hellbrügge T, Hrsg. Kinder ohne Bindung - Deprivation, Adoption und Psychotherapie. Stuttgart: Klett-Cotta;2006. S. 259-76.

130. Salzgeber J. Zum aktuellen Stand der PAS-Diskussion. ForumFamErbr. 2003;7(6):232-5.

131. Salzgeber J. Rezension von William Bernet: Parental Alienation, DSM-5 and ICD-11. Z Kindschaftsrecht Jugendhilfe. 2011;12:480-2.

132. Sauber SR. Differentiation between alienation, estrangement and bona fide abuse for the development of the reunification plan. Canadian Symposium for Parental Alienation Syndrome, Montreal, Dawson College. 2011.

133. Sauber SR. Reunification planning and therapy. In: Lorandos D, Bernet W, Sauber SR, Hrsg. Parental alienation the handbook for mental health and legal professionals. Springfield: C. C. Thomas;2013. S. 190-231.

134. Sauber SR, Worenklein A. Custody evaluations in alienation cases. In:BakerAJL, Sauber SR, Hrsg. Workingwith alienated children and families - a clinical guidebook. New York: Routledge; 2013. S. 47-70.

135. Siegel J, Langford J. MMPI-2 validity scales and suspected parental alienation syndrome. Am J Forensic Psychol. 1998;16(4):5-14.

136. von Staudingers J. Kommentar zum Bürgerlichen Gesetzbuch mit Einführungsgesetz und Nebengesetzen, Buch 4 Familienrecht $\S \S 1684-1717$ (ElterlicheSorge3-Umgangsrecht), Neubearbeitung: Coester, Rauscher, Salgo. Berlin: Sellier-deGruyter; 2014.

137. Steller M. Aussagepsychologie vor Gericht, Methodik und Probleme von Glaubwürdigkeitsgutachten mit Hinweisen auf die Wormser Missbrauchsprozesse. Recht Psychiatr. 1998;16(1):1-18.

138. Stephens R. The long history of PAS. 2016. http:// unknownmisandry.blogspot.com/2016/12/the-longhistory-of-pas-parental.html. Zugegriffen: 21. Febr. 2017, and http://disgustedwiththesystem.blogspot.com/2009/ 06/long-history- of-pas.html.

139. Tejedor Huerta A, Molina Bartumeus A, Orellana VN. PIVIP - Programa de Intervención para Victimas de Interferencias Parentales. Madrid:EOS; 2013.

140. Templer K, Matthewson M, Haines J, Cox G. Recommendations for best practice in response to parental alienation: findings from a systematic review. J Fam Ther. 2016; https:// doi.org/10.1111/1467-6427.12137.

141. Thaler-Singer M. Cults in our midst. The continuing fight against their hidden menace. San Fransico:Wiley; 2003.

142. von Uexküll Th. Psychosomatische Medizin. 5. Aufl. München, Wien, Baltimore: Urban \& Schwarzenberg; 2002.

143. Volbert R. Beurteilung von Aussagen über Traumata Erinnerungen und ihre psychologische Bewertung. Bern: Huber; 2004.
144. Wallerstein JS, Kelly JB. The effects of parental divorce: experiences of the child in later latency. Am J Orthopsychiatry. 1976;46:256-69.

145. Wallerstein JS, KellyJB. Surviving the breakup: how children and parents cope with divorce. New York: Basic Books; 1980.

146. Wallerstein J, Lewis JM, Blakeslee S. The unexpected legacy of divorce-the 25 yearlandmarkstudy. NewYork:Hyperion; 2000.

147. Warshak RA. Social science and parental alienation: examining the disputes and the evidence. In: Gardner RA, Sauber SR, Lorandos D, Hrsg. International handbook of parental alienation syndrome. Springfield: C. C. Thomas; 2006. S. 352-71.

148. Warshak RA. Family bridges: using insights from social science to reconnect parents and alienated children. Fam CourtRev. 2010a;48(1):48-80.

149. Warshak RA. Alienating audiences from innovation: the perils of polemics, ideology, and innuendo. Fam Court Rev. 2010b;48(1):153-63.

150. Warshak RA. Severe cases of parental alienation. In: Lorandos D, Bernet W, Sauber SR, Hrsg. Parental alienation - the handbook for mental health and legal professionals. Springfield: C. C. Thomas; 2013. S. 125-62.

151. Warshak RA. Ten parental alienation fallacies that compromise decisions in court and in therapy. Prof Psychol Res Pr. 2015a;46(4):235-49.

152. Warshak RA. Parental alienation: overview, management, intervention and practice tips. J Am Acad Matrim Lawyers. 2015b;28:181-248.

153. Warshak, RA. Family Bridges outcome study. Manuscript in preparation; 2018 (nach persönlicher Kommunikation mit Prof. Washakper E-mailvom31.01.2018)

154. Warshak RA, Otis MR. Helping alienated children with family bridges: practice, research, and the pursuit of "humbition.".Fam Court Rev. 2010;48(1):91-7.

155. Whitcombe S. Psychopathology and the conceptualisation of mental disorder: the debate around the inclusion of parental alienation in DSM-5. Counc Psychol Rev. 2013;28(3):6-18.

156. Woodall N, Woodall K. Understanding and working with the alienated child, family separation clinic London. 2014. www.familyseparationclinic.co.uk. Zugegriffen: 15. Dez. 2016.

157. Worenklein A. Moderate cases of parental alienation. In: Lorandos D, Bernet W, Sauber SR, Hrsg. Parental alienation - the handbook for mental health and legal professionals. Springfield: C. C. Thomas; 2013.S.97-194.

158. Zanarini MC, Williams AA, Lewis RE, Reich RB, Vera SC, Vera SC, Marino MF, Levin A, Yong L, Frankenburg FR. Reported pathological childhood experiences associated with the development of borderline personality disorder. Am J Psychiatry. 1997;154:1101-6.

Dr. med. Wilfrid von Boch-Galhau, Facharzt für Psychiatrie und Neurologie, Psychosomatische Medizin und Psychotherapiei.R.,Ehem. Mitglied des Interdisziplinären Arbeitskreises „Beratung bei Trennung und Scheidung“, Würzburg, Mitorganisator der internationalen Parental Alienation SyndromeKonferenz 2002, Frankfurt (Main), Mitglied der internationalen Parental Alienation Study Group (http://pasg.info). 